\title{
53rd ANNUAL SASKATCHEWAN CHRISTMAS BIRD COUNT — 1994
}

Compiled by Wayne C. Harris, Box 414, Raymore, Saskatchewan. S0A 3J0

With unseasonably warm weather during the entire count period (17 December 1994 through 2 January 1995) it seemed less like Christmas than it has for a number of years. The southwest portion of the province was completely without snow while other areas, even though snow covered, were loosing snow depth to the warm weather. The 88 counts completed this year were one less than last year and, in spite of the warm weather, the number of people participating dropped from 605 in 1993 to 597 this year. With the reduction in both the number of counts and participants a corresponding drop was inevitable in the number of hours accumulated - 1,006 compared to 1,120 last year.

\section{Weather and Coverage}

Table 1 summarizes the weather conditions reported on the counts. As indicated, the temperatures were unseasonably mild, with the average overnight low being $-10^{\circ} \mathrm{C}$ and the daytime highs averaged $-5^{\circ} \mathrm{C}$. Turtle Lake had the dubious honour of having the coldest night at $-30^{\circ} \mathrm{C}$ while Coronach basked in the mid-afternoon sun at $+12^{\circ} \mathrm{C}$. The heaviest snow cover was reported from the east side - Hudson Bay reported the most with almost a metre on the ground. Many locations were without any snow. The average snow depth for the entire province was only 11 $\mathrm{cm}$. Saskatchewan is noted for its windy weather but, as with everything else, the wind was a pleasant surprise - there was virtually none, with an overall average of only 8 53(2). June 1995 kmph, which by Saskatchewan standards is almost dead calm.

\section{The Birds}

There were 94 species recorded on count day, with an additional four species during the count period giving an overall total of 98 species, the same as last year. The results are compiled in Tables 3, 4 and 5. The total number of birds seen totalled 112,685 individuals, higher than the almost 100,00 seen in 1990. The most abundant species was again the Snow Bunting with a total of 28,730 individuals reported. Surprisingly, the second most common species was the Mallard $(26,168)$ which stayed in record numbers in the south where the lack of snow allowed them to continue to feed in the fields. House Sparrow $(18,077)$, Bohemian Waxwing $(11,382)$, Rock Dove (6,772), Canada Goose $(3,491)$, Black-billed Magpie $(3,319)$ and Black-capped Chickadee rounded out the list of species which exceeded 2,000 individuals on the counts.

Population trends of some of the most common species are provided in Table 7. The most substantial increases were for the semi-hardy species which linger longer when conditions are mild. Golden-crowned Kinglets experienced the biggest increase $(1,423 \%)$. This is most encouraging and continues the general upward trend in their numbers since the massive winter die off in the late 1970s. INext on the list was Mallard $(1,019 \%)$. Other species 
which fit the semi-hardy category and had large increases were Northern Flicker (430\%), Brown Creeper $(236 \%)$, American Tree Sparrow $(328 \%)$ and Red-winged Blackbird $(289 \%)$. One species which one would have expected to increase with the lack of snow is the Horned Lark, but it actually decreased. Whether the decrease is real or an artifact of the birds feeding in the open fields instead of on the roadsides, which is the case when the fields are covered in snow, remains to be seen.

Grouse populations generally improved with both Ruffed and Spruce Grouse continuing upwards on track with their normal cyclic trends. Northern Goshawks which normally parallel the grouse populations were very scarce. Forest owls were virtually non-existant, with only single Great Gray and Hawk Owls and no Barred, Boreal or Saw-whets.

The lack of cones on the spruce trees was undoubtedly a factor in the decline in numbers of Red-breasted Nuthatch and both crossbill species. White-winged Crossbills were not even found during count day and appear only as an additional species.

Of the winter finches, only Pine Grosbeaks were present in good numbers, showing an overall increase. Redpolls and Evening Grosbeaks were less common, particularly the redpolls (Hoary Redpolls were absent from counts completely).

\section{Rare Species}

For the second year in a row, no new species were added to the alltime list. Table 4 lists the least frequent species on the counts and the rarest species are all in this table. The first Willow Ptarmigan in a number of years were found on the
McMahon Lakes count. It is not that this species is necessarily rare but a fact that few counts are carried out far enough north to find this species. Of the truly rare, the Varied Thrushes found at both Fort Walsh and Whitewood rank among the best. The Rufous-sided Towhee found at Skull Creek was only the fourth count record and follows two separate occurrences from last year. American Goldfinches were found in both Prince Albert and Saskatoon.

House Finches were reported from five separate counts, phenomenal considering they were a new species only two years ago. It is becoming obvious that the cold extremes of our winters are not going to stop the spread of this species which has expanded rapidly westward from eastern North America in the past ten years.

Table 6 provides a summary of new high counts recorded for species during the 1994 count. The Mallard high was $50 \%$ higher than the previous with over 15,000 seen at the Cookson Reservoir on the Coronach count. This same count turned in new records for Gadwall (3) and American Coot (23). Both eagle species had new records with 79 Bald Eagles at Squaw Rapids (19 in one tree!!!) and 17 Golden Eagles on the Grassland National Park count. The 22 Northern Flickers at Saskatoon were more than twice the previous high and the 11 Pileated Woodpeckers at Kamsack almost three times the previous high. Finally, of note were the 393 Common Ravens at Hudson Bay, many of which were at the garbage dump.

\section{Count Areas and Participants}

Names of compilers are in italics. Number of participants in each count are in parentheses following the names. 
1. ABERNETHY-KATEPWA LAKE. Ron Bittner, Ron Hooper, Joy McKen, Wanda Steuck. (4)

2. ARMIT. Valeri Harris, Wayne Harris. (2)

3. ASSINIBOIA. Ed Bearss, Jack Burgeson, Alec Duxbury, Cecil Hayward, Marjorie Lamb, Nelson Lamb, Delmar Pettem, Wilf Prentice, Ken Schuweiler. (9)

4. BANGOR. Jean Hilton. (1)

5. BETHUNE. Doug and Vera Laing. (2)

6. BIGGAR. Roger Assailly, Guy Wapple, Morgan Wapple, Robert Wapple. (4)

7. BIRCH HILLS. Marg Mareschal, Moe Mareschal, Don Weidl. (3)

8. BROMHEAD. Martin Bailey, Carol Bjorklund. (2)

9. CANDLE LAKE. Burke Korol, Penny Pearse. (2)

10. CHRISTOPHER LAKE. Dorothy Bird. (1)

11. CLARK'S CROSSING. Barbara Brokx, Emile Brokx, Gail Bunt, Muriel Carlson, Adam Cooper, Don Doell, Bernie Gollop, Bob Johanson, Gerhard Lahey, Koji Maeda, Cliff Matthews, Mack Miller, Menno Nickel, Bruce Noton, Hilda Noton, Keith Pahl, Ann Pocknell, Wayne Shiels, Hilda Voth, Jim Wedgwood, Michael Williams, Jim Wood, Lois Wooding. (23)

12. CORONACH. Anne Harris, Valeri Harris, Wayne Harris. (3)

13. CRAVEN. Martin Bailey, Margaret Belcher, Andy Belyk, Al Binney, Betty Binnie, Carol Bjorklund, Doug Bradford, Dot Evans, Trevor Herriot, Phil Holloway, Phyllis Ilsley, Bob Kreba, Ferne Lawrence, Kevin Moore, Ron Myers, Bill Ogilvie, Curtis Pollack, Karyn Scalise. (18)

14. CROOKED LAKE. Bill Livesay, Mayta Livesay, Dorothy Skene, Ed Skene. (4)

15. CROOKED RIVER. Karl Mehler, Katherire Mehler, Kristine Mehler, Margaret Mehler, Morley Mehler, Phyllis Siemens. (6)

16. DILKE. Margaret Belcher, Brian McArton, Ken McArton, Bertha Wilson. (4)

17. DUVAL. Enid Cumming, George Herber, Linda Korytko, Kim McBride, lain Richardson, Lloyd Saul. (6)
18. EASTEND. Henri Lebastard. (1)

19. ENDEAVOUR. Norman Harris. (1)

20. FENTON. Carman Dodge. (1)

21. FIFE LAKE. Martin Myers, Robert Rafuse. (2)

22. FORT QU'APPELLE. Elizabeth Aitken, James Armstrong, Phyllis Bordass, William Bordass, Errol Cochrane, J. Connors, Anne Davies, Doug Evans, Doug Harman, Ronald Hooper, Lois Lamontagne, Vic Lamontagne, Maurice Lindgren, Jack Lowe, Don McDougall, Jean McKenna, Alan Mlazgar, Webb Palmer, Paul Paquin, Helen Reimer, Doreen Rowell, Lorne Rowell, Lloyd Talbot, Gus Vanderpolder. (24)

23. FORT WALSH. Paul Chytyk, Anne Harris, Valeri Harris, Wayne Harris, Burke Korol, Wilkes Parsonage, John Pollock, Matt Smith, Guy Wapple. (9)

24. GARDINER DAM. Paul Chytyk, Anne Harris, Valeri Harris, Wayne Harris, Ron Jensen, Burke Korol, John Pollock, Matt Smith, Guy Wapple, Roberi Wapple. (10)

25. GOOD SPIRIT LAKE. Bill Anaka, Joyce Anaka, Julia Wiwchar. (3)

26. GOVENLOCK. Paul Chytyk, Anne Harris, Valeri Harris, Wayne Harris, Burke Korol, John Pollock, Matt Smith, Guy Wapple. (8)

27. GRASSLANDS NATIONAL PARK. Paul Chytyk, Anne Harris, Valeri Harris, Wayne Harnis, John Pollock. (5)

28. GRASSLANDS NATIONAL PARK (NW). Pat Fargey, Carol Masecar, Florence Miller, Mike Wynn, Miriam Wynn, Wake Wynn. (6)

29. GRAYSON. Charles Helm, Karl Zimmer, Steven Zimmer. (3)

30. HANLEY. Caman Dodge, Keith Dodge Ken Dodge. (3)

31. HEPBURN. Phyllis Siemens. (1)

32. HUDSON BAY. Ian Daisley, John Daisley, Robert Daisley, Bruce Keyowski, Ryan Zens. (5)

33. HUMBOLDT. Ed Brockmeyer, Mike Volk. (2)

34. HUMBOLDT. (West). Bob Rafuse. (1)

35. INDIAN HEAD. Caroline Beatty, Jan Beattie, Denise Beaulieu, Margo 
Beaulieu, Vic Beaulieu, Irv Escott, David Gehl, Roberta Gehl, Gordon Howe, John Kort, Mary Kort, Dora Nichols, Lorne Scott, Fred Skinner, Anne Willerth, Gordon Willerth. (16)

36. KAMSACK. John Barisoff, George and Mary Bernard, Agnes Betz, Mable Buceuk, Lindee Duwores, Barb Elasser, Mrs. R.E. Elasser, Gordon Els, Jay Fry, Anita Klochko, Bill Koroluk, Joyce McLean, Dallas, Kelsey and Riley Rezansoff, Isabe/ Ritchie, David and Elsie Severson, John Solmon, Elenor Sookocheff, Phyllis Ward. (20)

37. KELVINGTON. Pat Finnie, Dianne Sloan, Marguerite Sloan. (3)

38. KENASTON. Lawrence Beckie. (1)

39. KILWINNING. Alan Daku, Ed Driver, Marg Driver. (3)

40. KINDERSLEY. Jean Harris, Keith Harris. (2)

41. KINLOCH. Don Forbes, Frank Gabriel Cliff Logan, Wilf Rodenberg. (4)

42. KUTAWAGAN LAKE. Wayne Hamis. (1)

43. LARONGE. Jim Paul, Lorie Ann Paul. (2)

44. LAST MOUNTAIN LAKE N.W.A. John Dunlop, Valeri Harris, Wayne Hams. (3)

45. LEADER (North). Daisy Meyers. (1)

46. LEADER (South). John Flood, John Rauch, Brenda Schnell, Susan Springett. (4)

47. LIVELONG. Sarah Pavka. (1)

48. LUSELAND. Kim Finley, Estelle Finley, Kerwin Finley, Bill Frey, Brent Honeker, Shirley Honeker, Wyatt Martfeld. (7)

49. MACDOWALL. Myron Barton. (1)

50. MATADOR. Cindy Romo, Jim Romo. (2)

51. MCMAHON LAKES. Daryl Johanneson, Dan Poirier. (2)

52. MEADOW LAKE. Bill Caldwell, Janet Caldwell, Betty Ann Golly, Byron Golly, Stuart Golly, Tyler Golly, Anne Sequin, Randy Sequin, Robbie Sequin, Bob Wilson. (7)

53. MELFORT (A). Frieda Markland. (1)

54. MELFORT (B). Phil Curry. (1)

55. MELVILLE. Donna Alberts, Marion MacLean, Jeanette Olson, Ross
Wotherspoon. (4)

56. MOOSE JAW. Andy and Vi Anderson, Edith Bell, Doug and Helen Brunsdon, Barry and Fern Dowse, Ed and Hartley Fredeen, Mary Jane Green, Al Gurnsey, Kerry Hanley, Eve King, Cy and Leith Knight, Gerald and Jean Lynn, Connie and Hugh Mclntyre, Helen and Peter Norys, Gavina Reekie, Ed Walker. (23)

57. MOOSE MOUNTAIN. Ray Belanger, Greg Bobbitt, Ross Douglas, Stewart Stairmand. (4)

58. NAICAM. Eldor Jensen, Ron Jensen. (2)

59. NICOLLE FLATS. Randi Edmonds, Alan Smith. (2)

60. PADDOCKWOOD. Blake and Stella Jones, Cliff Matthews. (3)

61. PIKE LAKE. Rich and Irene Barsky, Gail Bunt, Angeline Bernier, Kent and Laurel Brace, Wayne Cameron, Doug and Helen Campbell, Muriel Carlson, Betty Ann and Tom Dunlop, Paul Coutu, Sandra Flood, Mary Gilliland, Bernie Gollop, Mike Gollop, Logan and Marie Kennedy, Kay Kruger, Gerard Lahey, Joyce Manton, Menno Nickel, Keith Pahl, Gladys and Glen Pippin, Ken Pyette, Frank Roy, Monte and Pauline Sawyer, Michael Williams, Jim Wood, Lois Wooding, Nancy Young. (31)

62. PRINCE ALBERT (A). John Burt, Pamela Burt. (2)

63. PRINCE ALBERT (B). Jan Cote, Sherri Cote, Yvette Crane, Carman Dodge, Keith Dodge, Bebe Gilmour, Joe Graumans, Cam Harper, Lyle Hauser, Michael LeBlanc, Ian Monteith, Ted Snow, Troy Stimpson. (13)

64. PRINCE ALBERT NATIONAL PARK. Grace Carney, Susan Carr, Richard Cherepak, Emily and Greg Fenton, Shanna Frith, Vince Jones, Kurt Mazur, Grace McLeod, Bradley Muir, Adam and Samuel Pidwerbeski, Marg Tarleton, T.K. Tomlinson, Jeff, Libby and Nigel Weir. (17)

65. QU'APPELLE VALLEY DAM. Bob Plaster, Janice Plaster, Leah Plaster, Tyler Plaster, Frank Roy, Hilda Voth, Michael Williams. (7)

66. RAYMORE. Anne Harris, Valeri Harris, Wayne Harns, Sheila Lamont. (4)

67. REGINA. Sandy Ayer, Jessie Bailey, Martin Bailey, Margaret Belcher, Carol 
Bjorklund, Lionel Bonneville, Stephane Bonneville, Ray Christensen, Randi Edmonds, Robert Ewart, Sandra Ewart, Kay Ferguson, Mary Ferguson, Karen Herriot, Norman Herriot, Trevor Herriot, Dale Hjertaas, Estelle Hjertaas, Kerry Hjertaas, Paule Hjertaas, Wendy Horsefall, Phyllis Ilsley, Bob Kreba, Bob Luterbach, Bob MacFarlane, Lauren Mang, Ron Meyers, John Pollock, Gillian Richardson, Trevor Richardson, Karyn Scalise, Alan Smith, Frank Switzer. (33)

68. ROUND LAKE. Doug Francis, Joe and Laura Grimeau, Boyd Metzler, Lou Tremblay, Joyce Tremblay. (6)

\section{SALTCOATS. Robert Barnhart. (1)}

70. SASKATCHEWAN LANDING PROVINCIAL PARK. Peter Ashcroft, Wayne Harris. (2)

71. SASKATOON. Tony Allen, Juhachi Asai, Kengo Asai, Mika Asai, Sumiko Asai, Neil Beck, Beverly Beland, Orval Beland, Bob Besant, Joyce Besant, Bernard Bisha, Norma Lee Bisha, Carol Blenkin, Eveline Boudreau, Anna Braun, Gail Bunt, Muriel Carlson, Louise Cook, Leah Currie, Fran Eldridge, Peter Farrington, Martin Gerard, Silvia Gerard, Collen Gerwing, Marshall Gilliland, Mary Gilliland, Sean Gilliland, Bernie Gollop, Mike Gollop, Bob Green, May Haga, John Hanbidge, Darlene Hay, Jim Hay, Mary Houston, Stuart Houston, Peter Hull, Richard Kerbes, Gerard Lahey, Audrey McKibbon, Cathryn Miller, David Miller, Garth Nelson, John Nickel, Menno Nickel, Keith Pahl, James Parker, Orion Schille, Jacques Sirois, Jim Smart, Joe Sondershausen, Inge Tabel, Phil Taylor, Jeff Turple, Jim Wedgwood, George West, Michael Williams, Jim Wood, Stan Woynarski; Beaver Creek Nature Centre staff. (60)

72. SCOTT. Alec Elmquist, Guy Wapple, Robert Wapple. (3)

\section{SHAMROCK. Hugh Henry. (1)}

74. SKULL CREEK. Frank Ballauf, Erin Bennetto, Jim Bennetto, Ray Bennetto, Doris Bircham, Ralph Bircham, Ralph Drever, Bob Nyen, Harvey Wasilow, Mark Wasilow, Wade Wasilow, Robin Wolfater. (12)

75. SNOWDEN. Allan and Rita Birhenthai, Irene Hagel, Karen Hagel, Ralph and Penny Johnson, Violet Lien, Lorne and Sharon Lindberg, Verna Messer, Betty and Harold Pagan,
Edna and Percy Pagan, Bill and Vera Schemenauer, Harold and Irene Thompson, Les and Marlene White. (20)

76. SPALDING. Karen Durell, Larry Durell, Mark Durell, Michelle Durell, Velma Spizawka. (5)

77. SPINNEY HILL. Ed Driver, Marg Driver. (2)

78. SPRUCE HOME. Sharon and Tom Dice. (2)

79. SQUAW RAPIDS. Valeri Harris, Wayne Harris, Guy Wapple, Robert Wapple. (4)

80. SWIFT CURRENT. Evelyn Anderson, Peter Ashcroft, Lisa Dale, Hugh Henry, Ron Jensen, Arleyne Lawson, Doug Lawson, Laure Neish, Doris Thoreson, Neil Vandendort, Kaye Waters, John Weston, Pearl Weston. (13)

81. TISDALE. Joyce Mohr. (1)

82. TURTLE LAKE (A). Marie Robinson, Merle Robinson. (2)

83. TURTLE LAKE (B). Steve and Carol Burand, Muriel Carlson, Gil Heim, Marlene Kalanack, Hazel and Howard Newton, Marie and Merle Robinson, Clayton Thompson, Vicki Tollefson, Michael Williams. (12)

84. WAKAW LAKE. Alex Bergerman, Joe Bergerman, Roman Bergerman. (3)

85. WEYBURN. Leo Belanger, Ray Belanger, Greg Bobbitt, Jim Burge, Ross Douglas, Fred Garner, Dick Gutfriend, Grace Kurtz, Phil Layh, Ray Neville, Nick Postey, Keith Sakatch, Stew Stairmand, Doyle Thomas. (14)

86. WHITE BEAR. Daryl Jordheim, Floyd Jordheim, Ruby Jordheim, Sig Jordheim. (4)

87. WHITEWOOD. Cliff Ashfield, Pat Connolley, Mavis Kay, Boyd Metzler, John Pollock. (5)

88. YORKTON. Irma Esopenko, Warren Hjertaas, Geoff Rushowick, Patrick Rushowick, Dorothy Skene, Edward Skene. (6) 


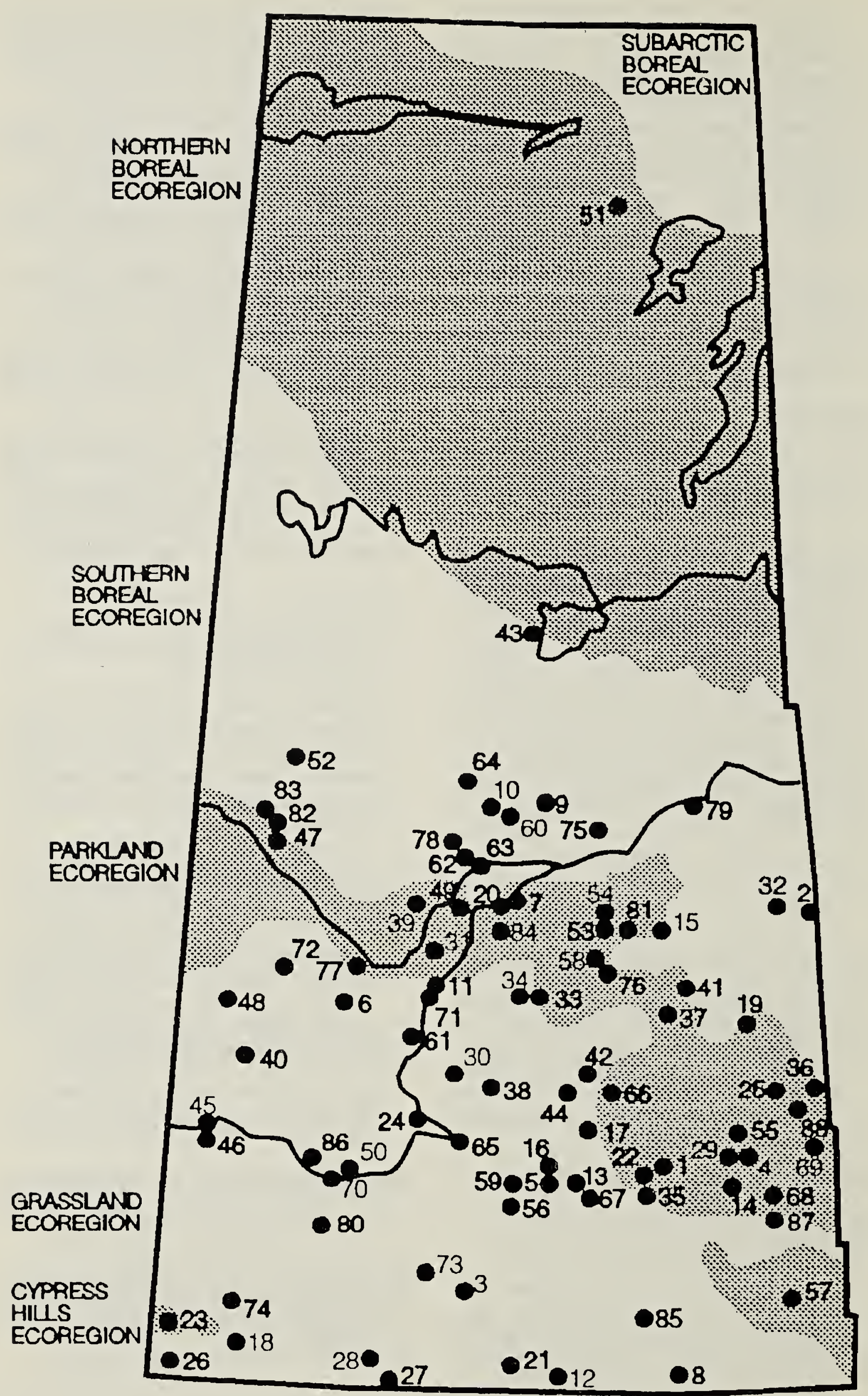

Figure 1. Location of 1994 counts (numbers correspond to locality names in Tables). 
Table 1. COUNT WEATHER CONDITIONS (Temperature ${ }^{\circ} \mathrm{C}$, Wind in $\mathrm{kmph}$ and Snow Cover in $\mathrm{cm}$ )

\begin{tabular}{|c|c|c|c|c|c|c|c|c|}
\hline LOCALITY & $\begin{array}{l}\text { Min. } \\
\text { Temp }\end{array}$ & $\begin{array}{l}\text { Max. } \\
\text { Temp }\end{array}$ & $\begin{array}{l}\text { Min. } \\
\text { Wind }\end{array}$ & $\begin{array}{l}\text { Max. } \\
\text { Wind }\end{array}$ & $\begin{array}{l}\text { Min. } \\
\text { Snow }\end{array}$ & $\begin{array}{l}\text { Max. } \\
\text { Snow }\end{array}$ & Sky A.M. & Sky P.M. \\
\hline $\begin{array}{l}\text { 1. ABERNETHY-KATEPWA } \\
\text { LAKE }\end{array}$ & -8 & +1 & 5 & 15 & 10 & 15 & clear & clear \\
\hline 2. ARMIT & -26 & -10 & 0 & 5 & 20 & 50 & overcast, light snow & $\begin{array}{l}\text { overcast, moderate } \\
\text { snow }\end{array}$ \\
\hline 3. ASSINIBOIA & +10 & +10 & 8 & 16 & 8 & 10 & partly cloudy & partly cloudy \\
\hline 4. BANGOR & -6 & 0 & 10 & 12 & 5 & 8 & mostly clear & mostly clear \\
\hline 5. BETHUNE & 0 & +2 & 10 & 15 & 5 & 10 & & clear \\
\hline 6. BIGGAR & -13 & -11 & 20 & 40 & 2 & 10 & partly cloudy, light fog & partly cloudy, light fog \\
\hline 7. BIRCH HILLS & -10 & -8 & 3 & 10 & 8 & 20 & $\begin{array}{l}\text { overcast, moderate fog. } \\
\text { light snow }\end{array}$ & overcast, moderate fog \\
\hline 8. BROMHEAD & -22 & -7 & 5 & 20 & 0 & 8 & clear & clear \\
\hline 9. CANDLE LAKE & -11 & -7 & 0 & 5 & 15 & 25 & partly cloudy & partly cloudy \\
\hline 10. CHRISTOPHER LAKE & -20 & -16 & 2 & 15 & 30 & 45 & clear & clear \\
\hline 11. CLARK'S CROSSING & -15 & -9 & 0 & 20 & 1 & 15 & overcast, moderate fog & mostly clear \\
\hline 12. CORONACH & -1 & +12 & 0 & 10 & 0 & 0 & clear & clear \\
\hline 13. CRAVEN & -12 & -8 & 5 & 10 & 7 & 15 & partly cloudy, fog & partly cloudy \\
\hline 14. CROOKED LAKE & -25 & -22 & 15 & 20 & 15 & 20 & clear & partly cloudy \\
\hline 15. CROOKED RIVER & -7 & +5 & 0 & 0 & 15 & 15 & clear & clear \\
\hline 16. DILKE & -19 & -17 & 20 & 30 & 0 & 10 & partly cloudy & clear \\
\hline 17. DUVAL & -12 & -6 & 0 & 12 & 0 & 10 & overcast & partly cloudy \\
\hline 18. EASTEND & -16 & +8 & 0 & 0 & 4 & 6 & mostly clear & partly cloudy \\
\hline 19. ENDEAVOUR & -10 & 0 & 0 & 0 & 12 & 15 & overcast, moderate fog & overcast, light fog \\
\hline 20. FENTON & -13 & -9 & 0 & 10 & 5 & 10 & $\begin{array}{l}\text { overcast, moderate fog. } \\
\text { moderate snow }\end{array}$ & overcast \\
\hline 21. FIFE LAKE & +5 & +10 & $\underline{0}$ & 5 & 0 & 0 & & clear \\
\hline 22. FORT QU'APPELLE & -12 & -10 & 0 & 5 & 8 & 10 & overcast, light fog & partly cloudy \\
\hline 23. FORT WALSH & -6 & -2 & 0 & 20 & 0 & 10 & mostly clear & mostly clear \\
\hline 24. GARDINER DAM & -10 & -4 & 0 & 20 & 0 & 5 & clear & partly cloudy \\
\hline 25. GOOD SPIRIT LAKE & -2 & +4 & 15 & 20 & 8 & 12 & mostly clear & mostly clear \\
\hline 26. GOVENLOCK & -1 & +10 & 0 & 5 & 0 & 0 & clear & clear \\
\hline 27. GRASSLANDS N.P. & -4 & +5 & 0 & 10 & 0 & 0 & mostly clear & mostly clear \\
\hline 28. GRASSLANDS N.P. (NW) & -2 & +3 & 0 & 10 & 0 & 0 & most!y clear & mostly clear \\
\hline 29. GRAYSON & +2 & +6 & 0 & 5 & 0 & 20 & partly cloudy & mostly clear \\
\hline 30. HANLEY & -10 & -2 & 0 & 5 & 0 & 5 & clear & clear \\
\hline 31. HEPBURN & -24 & -15 & 0 & 0 & 0 & 10 & clear & clear \\
\hline 32. HUDSON BAY & -10 & -20 & 10 & 25 & 60 & 90 & mostly clear & mostly clear \\
\hline 33. HUMBOLDT & -12 & -5 & 0 & 0 & 6 & 10 & heavy fog & heavy fog \\
\hline 34. HUMBOLDT $(\mathrm{W})$ & -18 & -15 & 0 & 0 & 15 & 20 & & $\begin{array}{l}\text { overcast, inoderate fog. } \\
\text { light snow }\end{array}$ \\
\hline 35. INDIAN HEAD & -20 & +3 & 0 & 20 & 5 & 10 & & \\
\hline 36. KAMSACK & -10 & -8 & 0 & 20 & 15 & 20 & overcast & partly cloudy \\
\hline 37. KELVINGTON & -23 & -20 & 0 & 0 & 30 & 45 & overcast, light fog & partly cloudy, light fog \\
\hline 38. KENASTON & -3 & +1 & 12 & 16 & 0 & 3 & clear & mostly clear \\
\hline 39. KILWINNING & -10 & -1 & $\underline{0}$ & 11 & 0 & 10 & fog & partly cloudy \\
\hline 40. KINDERSLEY & -10 & -10 & 0 & 1 & 0 & 1 & overcast, light fog & overcast, light fog \\
\hline 41. $\mathrm{KINLOCH}$ & 0 & +2 & 5 & 10 & 16 & 20 & mostly clear & mostly clear \\
\hline 42. KUTAWAGAN LAKE & -8 & -2 & 0 & 10 & 0 & 10 & overcast & overcast \\
\hline 43. LARONGE & -5 & -2 & 0 & 10 & 30 & 40 & overcast, light snow & overcast \\
\hline $\begin{array}{l}\text { 44. LAST MOUNTAIN LAKE } \\
\text { NW.A. }\end{array}$ & -10 & -2 & 0 & 15 & 0 & 12 & clear, light fog & overcast \\
\hline
\end{tabular}


Table 1. COUNT WEATHER CONDITIONS (continued)

\begin{tabular}{|c|c|c|c|c|c|c|c|c|}
\hline LOCALITY & $\begin{array}{l}\text { Min. } \\
\text { Temp }\end{array}$ & $\begin{array}{l}\text { Max. } \\
\text { Temp }\end{array}$ & $\begin{array}{l}\text { Min. } \\
\text { Wind } \\
\end{array}$ & $\begin{array}{l}\text { Max. } \\
\text { Wind }\end{array}$ & $\begin{array}{l}\text { Min. } \\
\text { Snow }\end{array}$ & $\begin{array}{l}\text { Max. } \\
\text { Snow }\end{array}$ & Sky A.M. & Sky P.M. \\
\hline 45. LEADER (North) & +1 & +4 & 2 & 5 & 0 & 0 & overcast & overcast \\
\hline 46. LEADER (South) & -10 & -10 & 0 & 0 & 0 & 3 & clear & clear \\
\hline 47. LIVELONG & -10 & +4 & 0 & 0 & 20 & 25 & clear & mostly clear \\
\hline 48. LUSELAND & -8 & -1 & 0 & 10 & 1 & 4 & overcast & partly cloudy \\
\hline 49. MACDOWALL & -22 & -20 & 12 & 15 & 18 & 25 & clear & clear \\
\hline 50. MATADOR & -7 & -5 & 0 & 30 & 0 & 0 & moderate fog & moderate fog \\
\hline 51. MCMAHON LAKES & -20 & -15 & 5 & 10 & 30 & 40 & partly cloudy & partly cloudy \\
\hline 52. MEADOW LAKE & -10 & -10 & 0 & 10 & 15 & 30 & clear & clear \\
\hline 53. MELFORT (A) & -7 & -4 & 2 & 5 & 10 & 15 & partly cloudy & mostly clear \\
\hline 54. MELFORT (B) & -18 & -14 & 8 & 15 & 30 & 40 & $\begin{array}{l}\text { overcast, moderate fog. } \\
\text { light snow }\end{array}$ & overcast, moderate fog \\
\hline 55. MELVILLE & -8 & -2 & 0 & 0 & 8 & 10 & mostly clear & mostly clear \\
\hline 56. MOOSE JAW & -3 & +8 & 0 & 9 & 0 & 0 & clear & overcast \\
\hline 57. MOOSE MOUNTAIN & -14 & +3 & 0 & 20 & 0 & 10 & clear & mostly clear \\
\hline 58. NAICAM & -4 & -4 & 0 & 15 & 10 & 50 & overcast & overcast \\
\hline 59. NICOLLE FLATS & -15 & -12 & 10 & 15 & 0 & 5 & mostly clear & mostly clear \\
\hline 60. PADDOCKWOOD & -8 & -8 & 10 & 15 & 20 & 20 & moderate fog & moderate fog \\
\hline 61. PIKE LAKE & -23 & -13 & 25 & 28 & 5 & 10 & mostly clear & partly cloudy \\
\hline 62. PRINCE ALBERT (A) & -24 & -17 & 1 & 2 & 35 & 60 & clear & clear \\
\hline 63. PRINCE ALBERT (B) & -12 & -7 & 0 & 10 & 10 & 20 & & \\
\hline 64. PRINCE ALBERT N.P. & -15 & -10 & 10 & 15 & 15 & 35 & mostly clear & mostly clear \\
\hline $\begin{array}{l}\text { 65. QU'APPELLE VALLEY } \\
\text { DAM }\end{array}$ & -11 & +4 & 0 & 10 & 0 & 8 & partly cloudy & mostly clear \\
\hline 66. RAYMORE & -5 & +4 & 0 & 10 & 0 & 15 & partly cloudy & mostly clear \\
\hline 67. REGINA & -10 & +1 & 5 & 15 & 10 & 25 & $\begin{array}{l}\text { partly cloudy, moderate } \\
\text { fog }\end{array}$ & overcast \\
\hline 68. ROUND LAKE & -8 & -1 & 0 & 0 & 0 & 12 & overcast, moderate fog & partly cloudy \\
\hline 69. SALTCOATS & -2 & +2 & 5 & 10 & 0 & 5 & partly cloudy & partly cloudy \\
\hline $\begin{array}{l}\text { 70. SASKATCHEWAN } \\
\text { LANDING P.P. }\end{array}$ & -8 & -4 & 20 & 30 & 0 & 0 & overcast & overcast \\
\hline 71. SASKATOON & -16 & -4 & 6 & 14 & 0 & 6 & mostly clear & overcast \\
\hline 72. SCOTT & -18 & -15 & 20 & 30 & 5 & 10 & partly cloudy & overcast, light snow \\
\hline 73. SHAMROCK & -6 & -6 & 5 & 35 & 0 & 0 & overcast & overcast \\
\hline 74. SKULL CREEK & +4 & +7 & 45 & 50 & 0 & 0 & overcast & overcast \\
\hline 75 SNOWDEN & -17 & -14 & 0 & 20 & 6 & 30 & $\begin{array}{l}\text { overcast, moderate } \\
\text { snow }\end{array}$ & overcast, light snow \\
\hline 76 SPALDING & -5 & -5 & 0 & 0 & 16 & 20 & overcast & overcast \\
\hline 77. SPINNEY HILL & -9 & -7 & 0 & 15 & 0 & 5 & mostly clear & mostly clear \\
\hline 78. SPRUCE HOME & -15 & -12 & 0 & 2 & 12 & 20 & partly cloudy & light fog \\
\hline 79. SQUAW RAPIDS & -11 & -2 & 0 & 10 & 25 & 30 & partly cloudy & partly cloudy \\
\hline 80. SWIFT CURRENT & -6 & -2 & 0 & 10 & 0 & 2 & overcast & partly cloudy \\
\hline 81. TISDALE & -10 & -8 & 0 & 0 & 10 & 15 & partly cloudy & \\
\hline 82. TURTLE LAKE (A) & -30 & -10 & 10 & 30 & 5 & 10 & mostly clear & mostly clear, light fog \\
\hline 83. TURTLE LAKE (B) & -14 & -12 & 5 & 12 & 10 & 30 & overcast, light snow & mostly dear, light snow \\
\hline 84. WAKAW LAKE & +1 & +4 & 5 & 10 & 5 & 8 & & partly cloudy \\
\hline 85. WEYBURN & -19 & -6 & 0 & 5 & 8 & 14 & overcast, moderate fog & overcast, moderate fog \\
\hline 86. WHITE BEAR & -8 & -6 & 0 & 2 & 0 & 0 & moderate fog & moderate fog \\
\hline 87. WHITEWOOD & -10 & 0 & 0 & 10 & 0 & 8 & clear & mostly clear \\
\hline 88. YORKTON & -12 & -10 & 15 & 40 & 5 & 25 & & \\
\hline
\end{tabular}




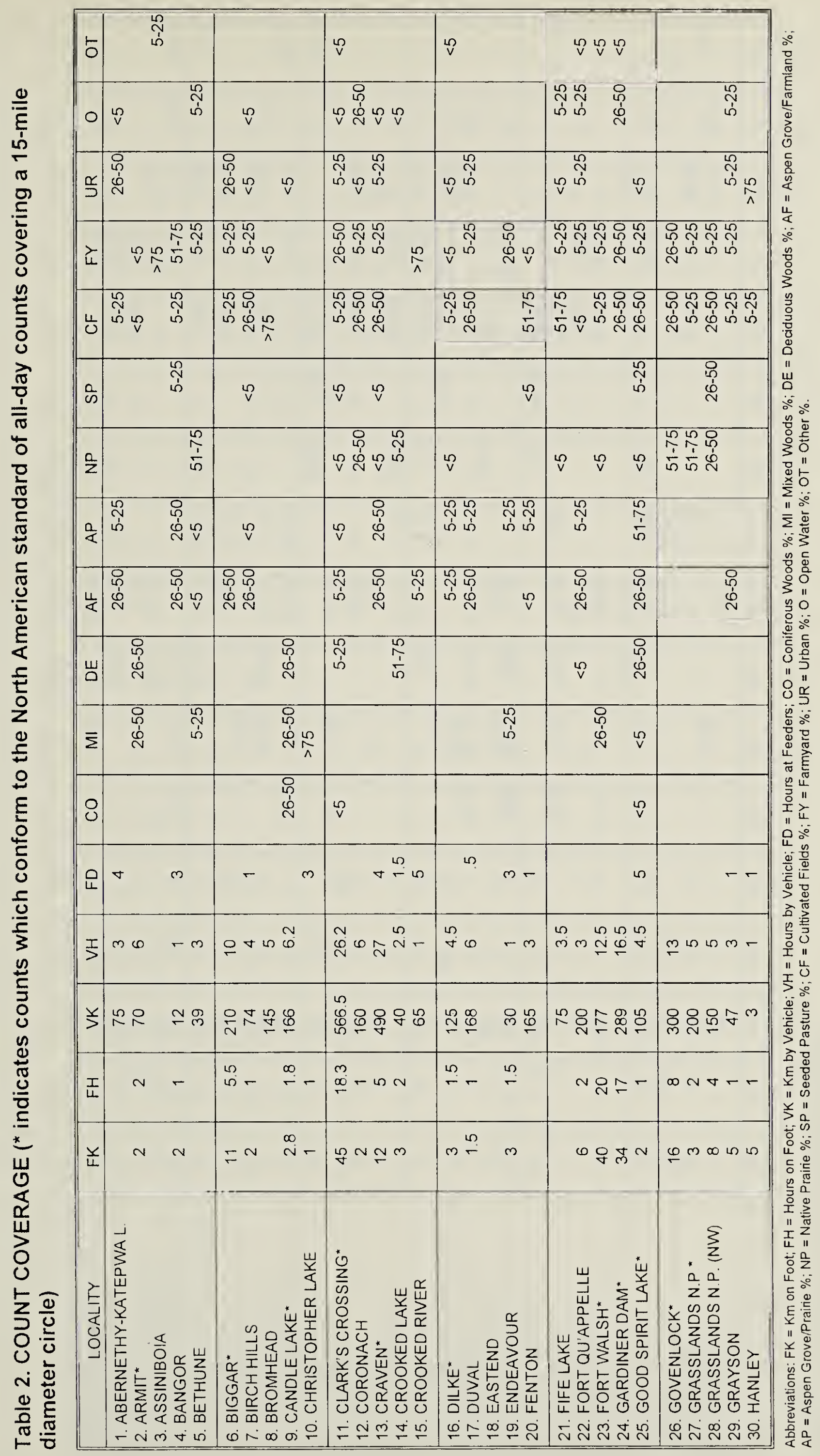




\begin{tabular}{|c|c|c|c|c|c|c|}
\hline - & $\begin{array}{l}\stackrel{\mathscr{N}}{\sim} \\
\stackrel{\sim}{n}\end{array}$ & & & $\begin{array}{l}\text { Oे } \\
\dot{q} \\
\text { N }\end{array}$ & 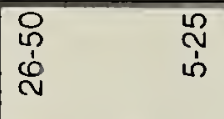 & 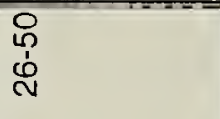 \\
\hline 0 & $\mathscr{\sim} \quad \stackrel{\sim}{\vee}$ & & ๗ & $\stackrel{\sim}{v}$ & & $\begin{array}{l}\stackrel{\mathscr{W}}{\omega} \\
\dot{\omega}\end{array}$ \\
\hline 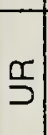 & $\begin{array}{ll}0 & \\
0 & \\
0 & \text { थ } \\
& v\end{array}$ & $\stackrel{\sim}{\vee} \mathscr{v}$ & 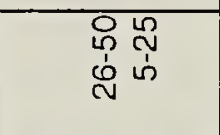 & ص & 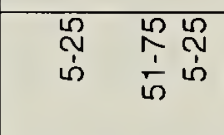 & \begin{tabular}{lll}
\multirow{N}{n}{} & \\
$\stackrel{\sim}{n}$ & L
\end{tabular} \\
\hline ז & 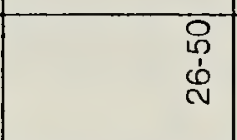 & 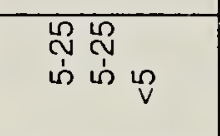 & 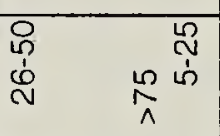 & $\begin{array}{ll} & \\
\stackrel{\sim}{\sim} & \stackrel{v}{V}\end{array}$ & $\begin{array}{l}\text { W్ } \\
\text { in }\end{array}$ & 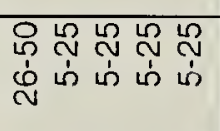 \\
\hline U. & $\frac{0}{\frac{1}{1}}$ & 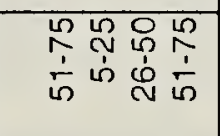 & 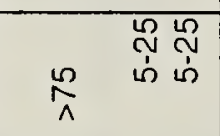 & 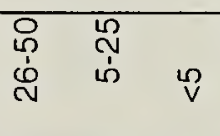 & 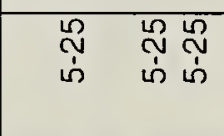 & 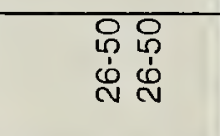 \\
\hline$\frac{a}{(5)}$ & & 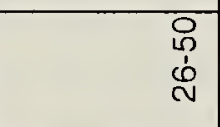 & $\begin{array}{l}\text { Wu } \\
\text { in } \\
\text { ñ }\end{array}$ & 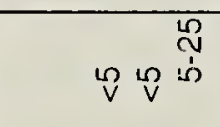 & & ? \\
\hline$\frac{a}{z}$ & $\stackrel{\sim}{\vee}$ & & 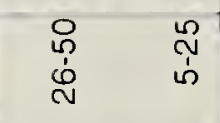 & 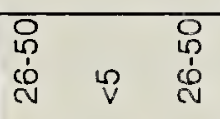 & $\stackrel{?}{v}$ & ? \\
\hline$\frac{n}{<}$ & 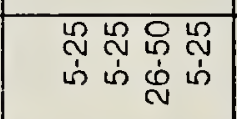 & $\begin{array}{l}\stackrel{n}{N} \\
\stackrel{n}{n}\end{array}$ & $\begin{array}{l}\stackrel{\text { Wu}}{\sim} \\
\dot{\omega}\end{array}$ & U & $\stackrel{\sim}{v}$ & $\begin{array}{l}\stackrel{n}{N} \\
\text { in }\end{array}$ \\
\hline$\frac{4}{4}$ & 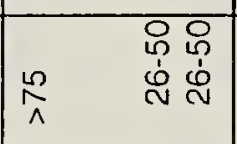 & 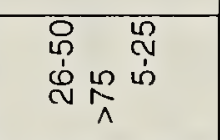 & $\begin{array}{l}\text { Wू } \\
\stackrel{W}{\omega} \\
\text { in }\end{array}$ & 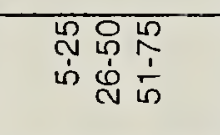 & 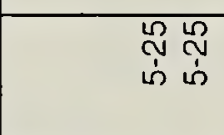 & 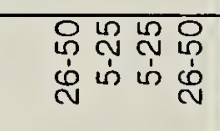 \\
\hline 㟔 & & 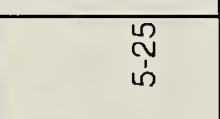 & $\begin{array}{ll}\stackrel{\mathscr{N}}{N} \\
\text { in }\end{array}$ & & 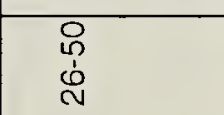 & $\begin{array}{ll}0 & 0 \\
0 & 0 \\
0 & 0 \\
0 & N\end{array}$ \\
\hline $\bar{\Sigma}$ & $\begin{array}{l}0 \\
0 \\
0 \\
c\end{array}$ & $\begin{array}{l}\stackrel{\text { ñ }}{\sim} \\
\text { in }\end{array}$ & 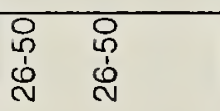 & 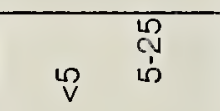 & 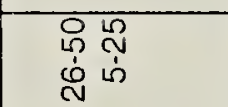 & $\begin{array}{l}\text { Wू } \\
\text { in } \\
\text { in }\end{array}$ \\
\hline 8 & & $\begin{array}{l}\stackrel{W}{W} \\
\dot{i n}\end{array}$ & $\begin{array}{ll}\stackrel{\mathscr{N}}{N} & \stackrel{\sim}{N} \\
\dot{\omega} & \dot{\omega}\end{array}$ & $\stackrel{\sim}{v}$ & $\begin{array}{l}\frac{5}{2} \\
\frac{1}{n}\end{array}$ & \\
\hline 인 & $-\quad-\varphi$ & $v_{-} \quad-$ & $\infty \quad-\infty N$ & $v \nabla N-$ & $\forall \sim \sim \sim$ & m \\
\hline$\frac{T}{>}$ & 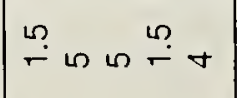 & $\mathrm{N}$ เ & $N \forall \forall \underset{I}{\infty} v$ & $\forall N$ « m & ๓யா & $\stackrel{\sim}{\forall} \forall \forall \omega \mathrm{m}$ \\
\hline$\stackrel{Y}{\zeta}$ & 음윳ㅇㅇㅇㅛ & 등 요용요 & 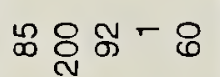 & 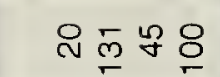 & 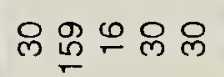 & ติ \\
\hline T & $-\stackrel{\sim}{\sim} \sigma$ & - & $\stackrel{n}{-}--+$ & $m \sigma d$ & $m \sim \sim \sim-$ & $\infty \stackrel{n}{\stackrel{n}{\longrightarrow}}-\infty$ \\
\hline ̌ㅏㄴ & 음 & --- & $\stackrel{n}{N}-m \quad 0$ & ก & $m \sim \operatorname{son}^{\circ} \sim$ & $\stackrel{n}{\infty}$ \\
\hline 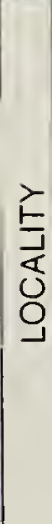 & 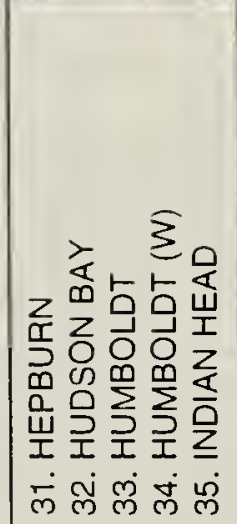 & 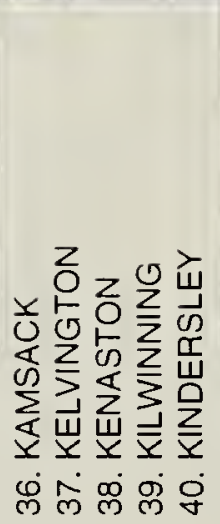 & 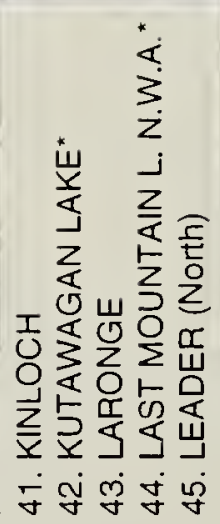 & 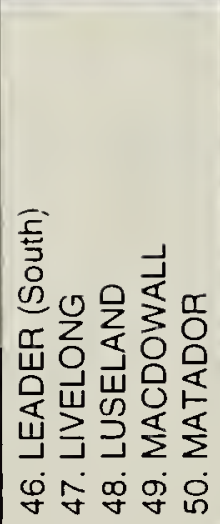 & 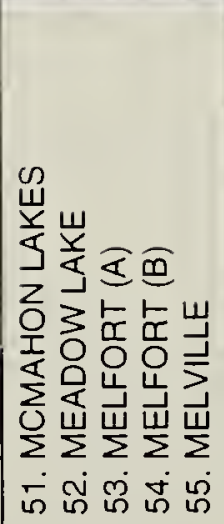 & 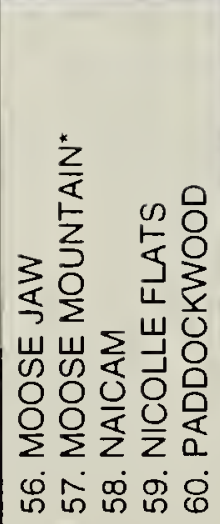 \\
\hline
\end{tabular}




\begin{tabular}{|c|c|c|c|c|c|c|}
\hline 5 & & $\stackrel{v}{\vee}$ & & & & 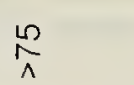 \\
\hline 0 & $\mathscr{v} \vartheta$ & 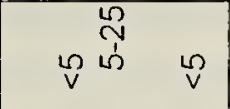 & & $\stackrel{v}{v} v$ & 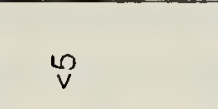 & \begin{tabular}{l}
0 \\
0 \\
0 \\
\multirow{2}{*}{}
\end{tabular} \\
\hline$\frac{x}{5}$ & 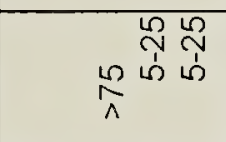 & 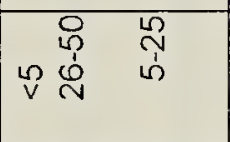 & 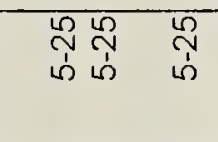 & 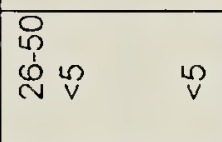 & $\stackrel{\mathscr{N}}{\stackrel{\mathscr{N}}{N}}$ & $\begin{array}{l}\text { W } 0 \\
\text { in } \\
\text { in } \\
\text { d }\end{array}$ \\
\hline 光 & 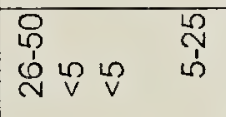 & 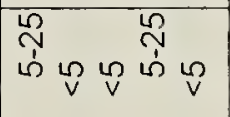 & 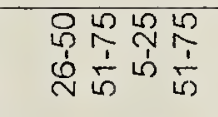 & 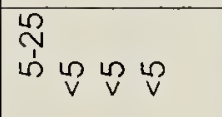 & 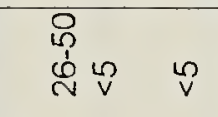 & 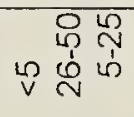 \\
\hline$\stackrel{4}{0}$ & 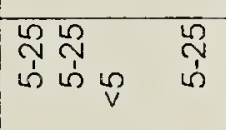 & 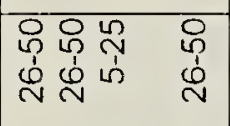 & 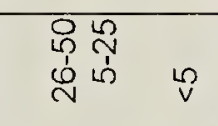 & 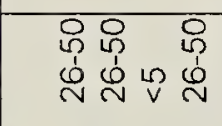 & 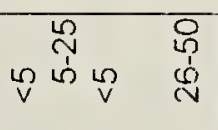 & W \\
\hline$\frac{2}{\infty}$ & $\stackrel{\mathscr{P}}{\mathscr{V}} \quad \stackrel{\sim}{V}$ & $\stackrel{\sim}{v}$ & & ? & $\stackrel{n}{v}$ & \\
\hline$\frac{a}{z}$ & 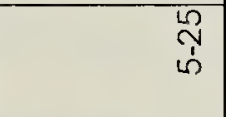 & $\vartheta \quad \stackrel{\frac{L}{5}}{\frac{1}{5}}$ & $\stackrel{\mathscr{N}}{\sim}$ & v $\stackrel{\sim}{\sim}$ & 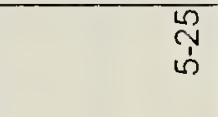 & \\
\hline$\frac{a}{<}$ & 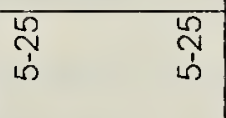 & 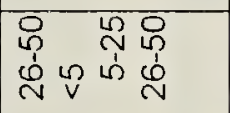 & $\begin{array}{l}\stackrel{W}{N} \\
\dot{\omega}\end{array}$ & $\stackrel{?}{v}$ & $\begin{array}{l}\text { W } \\
\text { is }\end{array}$ & \\
\hline$\frac{1}{\alpha}$ & 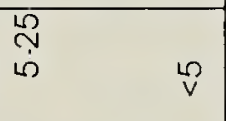 & 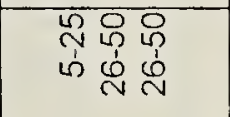 & 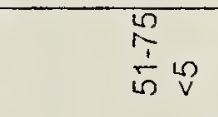 & 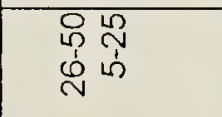 & 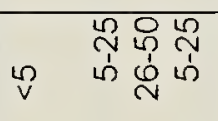 & $\begin{array}{l}\text { 员 } \\
\text { d. } \\
\text { N }\end{array}$ \\
\hline & 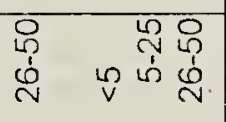 & v & & $\begin{array}{ll}0 & \omega \\
0 & N \\
0 & i \\
N & \text { in }\end{array}$ & 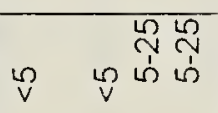 & \\
\hline $\bar{\Sigma}$ & 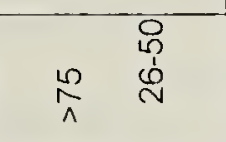 & & v & 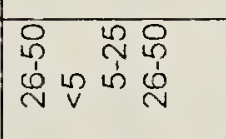 & 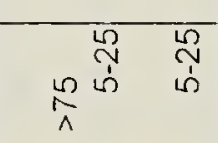 & \\
\hline 8 & $\underset{v}{\stackrel{i}{N}}$ & & & $\begin{array}{l}\stackrel{i}{N} \\
v \\
v\end{array}$ & 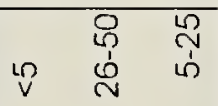 & \\
\hline 인 & $\stackrel{\sim}{\sim} \quad-$ & $-6 r$ & 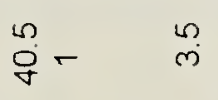 & $m \sim \stackrel{\infty}{\sigma}$ & - & $\stackrel{n}{\sim}$ \\
\hline$\frac{I}{S}$ & 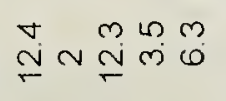 & $\infty \stackrel{n}{m} \forall \omega \theta$ & $\stackrel{\infty}{\forall} \wedge m \forall \stackrel{n}{\forall}$ & MONEF & $-\quad \sim \stackrel{m}{i}$ & $n^{n}$ \\
\hline$\stackrel{y}{>}$ & 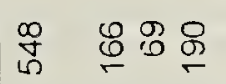 & 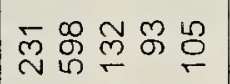 & $\stackrel{\overbrace{}}{\Omega}$ & চ 맘 & $\mathbb{N}_{N}^{2} \stackrel{\infty}{\sim}$ & $\stackrel{\infty}{\sim} \underset{v}{q}$ \\
\hline I & 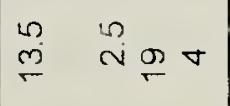 & $\nabla \underset{\sim}{*} N N-$ & 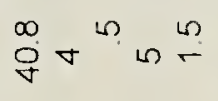 & $-\stackrel{\sim}{\infty}-\infty$ & $\alpha \stackrel{m}{r}$ & $r^{n} m$ \\
\hline 希 & 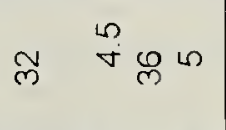 & $=F_{\infty}^{\infty} \backsim \sim N$ & 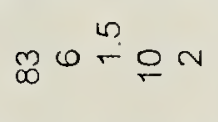 & $m^{n} m \approx N$ & $\begin{array}{r}n \\
\sigma \quad 0\end{array}$ & $\Delta$ \\
\hline $\begin{array}{l}\text { ¿ } \\
\bar{\delta} \\
\text { O }\end{array}$ & 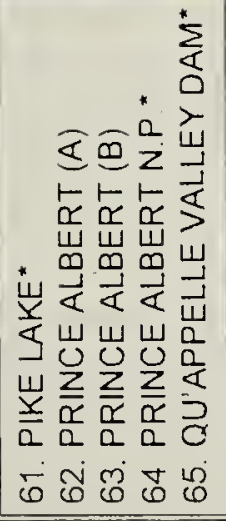 & 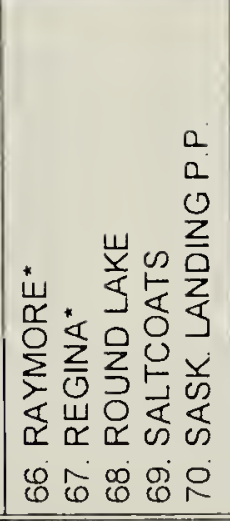 & 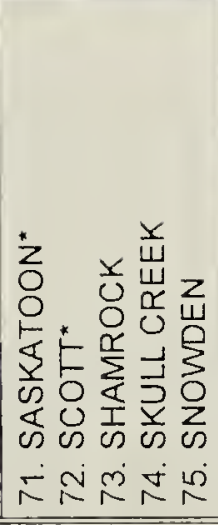 & 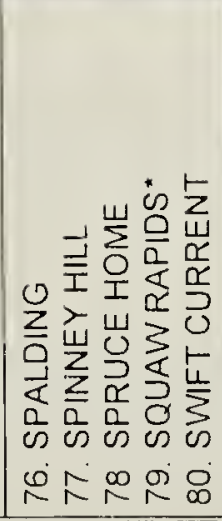 & 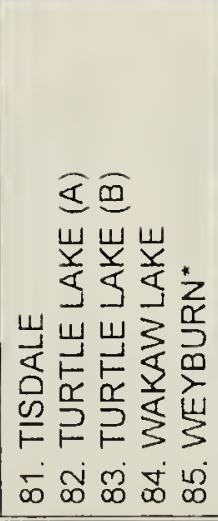 & 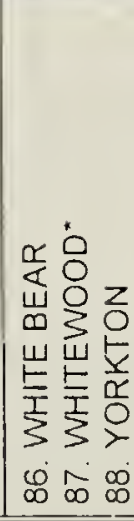 \\
\hline
\end{tabular}


Table 3-1. SPECIES RECORDED FROM MORE THAN FIVE LOCALITIES $(+=$ species seen during the count period but not on count day)

\begin{tabular}{|c|c|c|c|c|c|c|c|c|c|c|c|c|}
\hline & & & & & & & LOCAT & ION AN & D DATE & & & \\
\hline 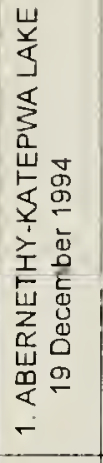 & 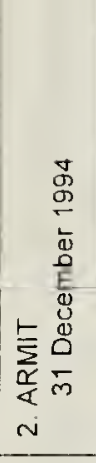 & 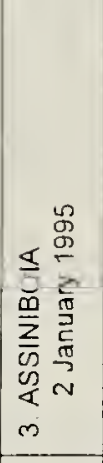 & 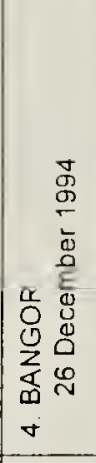 & 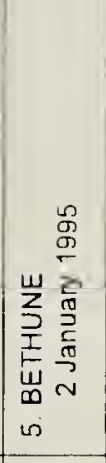 & 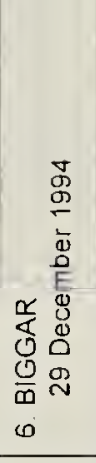 & 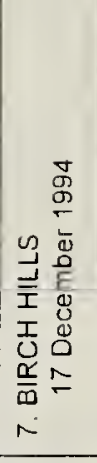 & 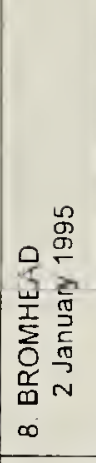 & 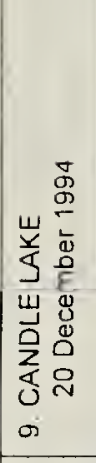 & 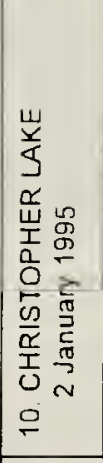 & 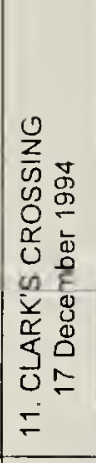 & 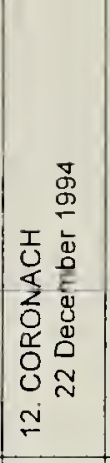 & SPECIES \\
\hline+ & & & & & & & & & & 40 & $\begin{array}{r}2760 \\
15150 \\
1 \\
1\end{array}$ & $\begin{array}{l}\text { CANADA GOOSE } \\
\text { MALLARD } \\
\text { COMMON GOLDENEYE } \\
\text { BALD EAGLE } \\
\text { NORTHERN GOSHAWK }\end{array}$ \\
\hline & & & & & 1 & & & & & & 9 & $\begin{array}{l}\text { ROUGH-LEGGED HAWK } \\
\text { GOLDEN EAGLE } \\
\text { MERLIN } \\
\text { GYRFALCON } \\
\text { PRAIRIE FALCON }\end{array}$ \\
\hline $\begin{array}{l}5 \\
3\end{array}$ & 7 & $\begin{array}{r}63 \\
5 \\
22\end{array}$ & $\begin{array}{r}2 \\
21\end{array}$ & 2 & $\begin{array}{r}2 \\
\\
3 \\
59 \\
\end{array}$ & $\begin{array}{r}27 \\
1 \\
34 \\
\end{array}$ & 8 & 3 & & 158 & 61 & $\begin{array}{l}\text { GRAY PARTRIDGE } \\
\text { RING-NECKED PHEASANT } \\
\text { RUFFED GROUSE } \\
\text { SHARP-TAILED GROUSE } \\
\text { ROCK DOVE } \\
\end{array}$ \\
\hline+ & $\begin{array}{l}1 \\
4 \\
2\end{array}$ & $\begin{array}{l}8 \\
4\end{array}$ & $\begin{array}{l}2 \\
2\end{array}$ & 1 & $\begin{array}{l}+ \\
1 \\
1 \\
1\end{array}$ & $\begin{array}{l}+ \\
2 \\
1 \\
1\end{array}$ & $\begin{array}{l}3 \\
1\end{array}$ & $\begin{array}{l}1 \\
4\end{array}$ & $\begin{array}{l}2 \\
3\end{array}$ & $\begin{array}{l}1 \\
4 \\
3 \\
4\end{array}$ & 1 & $\begin{array}{l}\text { GREAT HORNED OWL } \\
\text { SNOWY OWL } \\
\text { DOWNY WOODDPECKER } \\
\text { HAIRY WOODPECKER } \\
\text { THREE-TOED WOODPECKER }\end{array}$ \\
\hline 1 & $\begin{array}{r}18 \\
1 \\
\end{array}$ & & 1 & & \pm & 7 & 19 & $\begin{array}{r}21 \\
6 \\
\end{array}$ & $\begin{array}{l}3 \\
2 \\
\end{array}$ & & 1 & $\begin{array}{l}\text { NORTHERN FLICKER } \\
\text { PILEATED WOODPECKER } \\
\text { HORNED LARK } \\
\text { GRAY JAY } \\
\text { BLUE JAY } \\
\end{array}$ \\
\hline $\begin{array}{r}6 \\
14\end{array}$ & $\begin{array}{r}13 \\
82 \\
7\end{array}$ & 38 & $\begin{array}{l}3 \\
3 \\
8\end{array}$ & 3 & $\begin{array}{r}101 \\
8 \\
16 \\
2\end{array}$ & $\begin{array}{r}15 \\
7 \\
28\end{array}$ & 5 & $\begin{array}{r}21 \\
111 \\
40 \\
31 \\
1\end{array}$ & $\begin{array}{r}2 \\
25 \\
10 \\
2 \\
\end{array}$ & $\begin{array}{r}229 \\
56\end{array}$ & 7 & $\begin{array}{l}\text { BLACK-BILLED MAGPIE } \\
\text { COMMON RAVEN } \\
\text { BLACK-CAPPED CHICKADEE } \\
\text { BOREAL CHICKADEE } \\
\text { RED-BREASTED NUTHATCH }\end{array}$ \\
\hline 15 & & 6 & 30 & & $\begin{array}{r}2 \\
+ \\
773 \\
2 \\
\end{array}$ & 95 & & & 2 & $\begin{array}{r}+ \\
305\end{array}$ & & $\begin{array}{l}\text { WHITE-BREASTED NUTHATCH } \\
\text { GOLDEN-CROWNED KINGLET } \\
\text { AMERICAN ROBIN } \\
\text { BOHEMIAN WAXWING } \\
\text { CEDAR WAXWING } \\
\end{array}$ \\
\hline $\begin{array}{r}1 \\
+ \\
\end{array}$ & 335 & 25 & 50 & & 541 & 173 & 262 & & + & $\begin{array}{r}1 \\
51 \\
1 \\
274 \\
\end{array}$ & & $\begin{array}{l}\text { NORTHERN SHRIKE } \\
\text { EUROPEAN STARLING } \\
\text { HARRIS' SPARROW } \\
\text { DARK-EYED JUNCO } \\
\text { SNOWBUNTING } \\
\end{array}$ \\
\hline 43 & $\begin{array}{l}31 \\
45 \\
\end{array}$ & 350 & 8 & 100 & $\begin{array}{r}1 \\
+ \\
12\end{array}$ & $\begin{array}{r}12 \\
158 \\
\end{array}$ & 175 & 14 & 18 & $\begin{array}{r}1 \\
38 \\
19\end{array}$ & 110 & $\begin{array}{l}\text { FUST ELACKEIRC } \\
\text { PINE GROSBEAK } \\
\text { COMMON REDPOLL } \\
\text { PINE SISKIN } \\
\text { EVENING GROSBEAK } \\
\text { HOUSE SPARROW }\end{array}$ \\
\hline 10 & 16 & 9 & 13 & 6 & 20 & 19 & 9 & 13 & 11 & 19 & 14 & NO. SPECIES COUNT DAY \\
\hline 13 & 16 & 9 & 13 & 7 & 24 & 20 & 9 & 13 & 14 & 20 & 14 & NO. SPECIES CO!:NT PERIOD \\
\hline 91 & 591 & 521 & 138 & 116 & 2189 & 576 & 510 & 260 & 99 & 2600 & 18131 & NO. INDIV COUNT DAY \\
\hline 0 & 1 & 0 & 0 & 0 & 0 & 1 & 35 & 4 & 0 & 0 & 30 & NO. INDIV. IN TABLE $4 \& 5$ \\
\hline 0 & 1 & 0 & 0 & 0 & 0 & 1 & 1 & 1 & 1 & & & NO. SPECIES IN TABLE 485 \\
\hline
\end{tabular}


Table 3-2. SPECIES RECORDED FROM MORE THAN FIVE LOCALITIES $(+=$ species seen during the count period but not on count day)

\begin{tabular}{|c|c|c|c|c|c|c|c|c|c|c|c|c|}
\hline & & & LOCATI & ION ANL & D DATE & & & & & & & \\
\hline SPECIES & 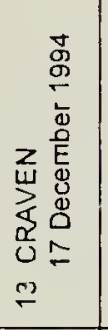 & 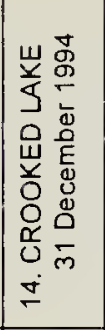 & 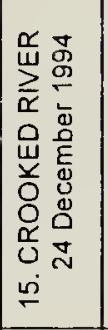 & 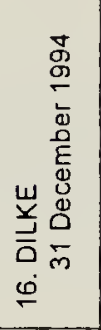 & 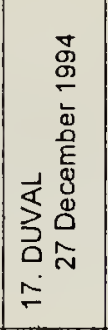 & 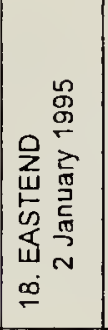 & 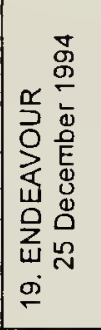 & 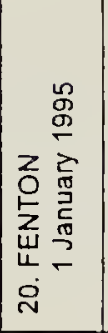 & 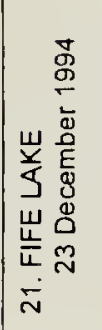 & 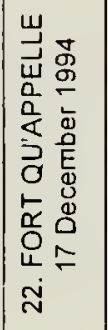 & 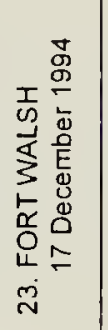 & 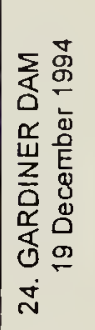 \\
\hline $\begin{array}{l}\text { CANADA GOOSE } \\
\text { MALLARD } \\
\text { COMMON GOLDENEYE } \\
\text { BALD EAGLE } \\
\text { NORTHERN GOSHAWK }\end{array}$ & & $\begin{array}{l}2 \\
2\end{array}$ & & & & & + & & $\begin{array}{r}500 \\
5000 \\
4 \\
+ \\
\end{array}$ & $\begin{array}{r}1 \\
45 \\
5 \\
2\end{array}$ & $\begin{array}{r}4 \\
10 \\
1 \\
\end{array}$ & $\begin{array}{r}15 \\
5722 \\
115 \\
31\end{array}$ \\
\hline $\begin{array}{l}\text { ROUGH-LEGGED HAWK } \\
\text { GOLDEN EAGLE } \\
\text { MERLIN } \\
\text { GYRFALCON } \\
\text { PRAIRIE FALCON }\end{array}$ & & & & & 1 & & & & $\begin{array}{r}+ \\
8 \\
+\end{array}$ & + & $\begin{array}{l}3 \\
2 \\
1\end{array}$ & $\begin{array}{l}3 \\
2\end{array}$ \\
\hline $\begin{array}{l}\text { GRAY PARTRIDGE } \\
\text { RING-NECKED PHEASANT } \\
\text { RUFFED GROUSE } \\
\text { SHARP-TAILED GROUSE } \\
\text { ROCK DOVE }\end{array}$ & $\begin{array}{l}13 \\
53 \\
\end{array}$ & 1 & 10 & $\begin{array}{r}+ \\
17 \\
\end{array}$ & $\begin{array}{r}2 \\
21 \\
\end{array}$ & $\begin{array}{l}4 \\
6\end{array}$ & + & & $\begin{array}{r}14 \\
1\end{array}$ & $\begin{array}{r}8 \\
+ \\
4 \\
\end{array}$ & $\begin{array}{l}8 \\
4 \\
1 \\
2 \\
1 \\
\end{array}$ & $\begin{array}{r}+ \\
29 \\
179 \\
\end{array}$ \\
\hline $\begin{array}{l}\text { GREAT HORNED OWL } \\
\text { SNOWY OWL } \\
\text { DOWNY WOODDPECKER } \\
\text { HAIRY WOODPECKER } \\
\text { THREE-TOED WOODPECKER }\end{array}$ & $\begin{array}{l}4 \\
3\end{array}$ & $\begin{array}{l}3 \\
2\end{array}$ & $\begin{array}{l}1 \\
3 \\
2 \\
2\end{array}$ & $\begin{array}{l}+ \\
+ \\
1\end{array}$ & $\begin{array}{l}2 \\
+ \\
1 \\
1\end{array}$ & $\begin{array}{l}2 \\
4 \\
2\end{array}$ & 3 & $\begin{array}{l}1 \\
1 \\
2 \\
3\end{array}$ & 1 & $\begin{array}{r}+ \\
11 \\
8\end{array}$ & $\begin{array}{l}4 \\
2 \\
2 \\
1 \\
\end{array}$ & $\begin{array}{l}3 \\
5 \\
3 \\
4\end{array}$ \\
\hline $\begin{array}{l}\text { NORTHERN FLICKER } \\
\text { PILEATED WOODPECKER } \\
\text { HORNED LARK } \\
\text { GRAY JAY } \\
\text { BLUE JAY } \\
\end{array}$ & $\begin{array}{l}11 \\
12 \\
\end{array}$ & 2 & & 18 & 3 & $\begin{array}{r}18 \\
6 \\
\end{array}$ & $\begin{array}{l}3 \\
2 \\
\end{array}$ & 1. & 13 & 14 & & $\begin{array}{l}2 \\
6 \\
\end{array}$ \\
\hline $\begin{array}{l}\text { BLACK-BILLED MAGPIE } \\
\text { COMMON RAVEN } \\
\text { BLACK-CAPPED CHICKADEE } \\
\text { BOREAL CHICKADEE } \\
\text { RED-BREASTED NUTHATCH }\end{array}$ & $\begin{array}{r}150 \\
54\end{array}$ & $\begin{array}{r}17 \\
2 \\
39\end{array}$ & $\begin{array}{l}1 \\
1 \\
7\end{array}$ & 20 & $\begin{array}{r}21 \\
1 \\
15\end{array}$ & $\begin{array}{c}18 \\
19\end{array}$ & $\begin{array}{l}21 \\
50 \\
22\end{array}$ & $\begin{array}{r}6 \\
2 \\
12\end{array}$ & 4 & $\begin{array}{r}27 \\
5 \\
126\end{array}$ & $\begin{array}{l}98 \\
77\end{array}$ & $\begin{array}{r}124 \\
16\end{array}$ \\
\hline $\begin{array}{l}\text { WHITE-BREASTED NUTHATCH } \\
\text { GOLDEN-CROWNED KINGLET } \\
\text { AMERICAN ROBIN } \\
\text { BOHEMIAN WAXWING } \\
\text { CEDAR WAXWING }\end{array}$ & $\begin{array}{r}6 \\
2 \\
8 \\
35 \\
49 \\
\end{array}$ & $\begin{array}{l}27 \\
16 \\
\end{array}$ & & 15 & & 10 & + & + & + & $\begin{array}{r}19 \\
+ \\
143 \\
+ \\
\end{array}$ & $\begin{array}{r}4 \\
12 \\
3\end{array}$ & 129 \\
\hline $\begin{array}{l}\text { NORTHERN SHRIKE } \\
\text { EUROPEAN STARLING } \\
\text { HARRIS' SPARROW } \\
\text { DARK-EYED JUNCO } \\
\text { SNOW BUNTING } \\
\end{array}$ & 142 & 3 & & + & 25 & & 75 & 130 & + & $\begin{array}{r}1 \\
20 \\
2 \\
83 \\
\end{array}$ & $\begin{array}{r}6 \\
3 \\
41 \\
\end{array}$ & $\begin{array}{r}58 \\
40 \\
\end{array}$ \\
\hline $\begin{array}{l}\text { RUSTY BLACKBIRD } \\
\text { PINE GROSBEAK } \\
\text { COMMON REDPOLL } \\
\text { PINE SISKIN } \\
\text { EVENING GROSBEAK } \\
\text { HOUSE SPARROW }\end{array}$ & 565 & & $\begin{array}{l}16 \\
20 \\
\end{array}$ & 69 & $\begin{array}{r}+ \\
373 \\
\end{array}$ & 100 & $\begin{array}{l}36 \\
13 \\
\end{array}$ & 12 & & $\begin{array}{r}2 \\
+ \\
+ \\
+ \\
+ \\
401 \\
\end{array}$ & \begin{tabular}{r|}
7 \\
90 \\
1 \\
131 \\
\end{tabular} & 1051 \\
\hline NO SPECIES COUNT DAY & 20 & 17 & 11 & 7 & 12 & 12 & 14 & 10 & 11 & 27 & 33 & 29 \\
\hline NO. SPECIES COUNT PERIOD & 20 & 17 & 11 & 14 & 14 & 12 & 17 & 11 & 26 & 37 & 33 & 30 \\
\hline NO. INDIV COINT DAY & 1120 & 127 & 78 & 143 & 466 & 192 & 243 & 170 & 5549 & 944 & 551 & 7763 \\
\hline NO. INDIV. IN TABLE 49.5 & 2 & 7 & 0 & 0 & 0 & 0 & 0 & 0 & 4 & 17 & 32 & 217 \\
\hline NO. SPECIES IN TABIE $₫ \& 5$ & 1. & 3 & 0 & 0 & 0 & 0 & 0 & 0 & 7 & 8 & 6 & 7 \\
\hline
\end{tabular}




\begin{tabular}{|c|c|c|c|c|c|c|c|c|c|c|c|c|}
\hline \multirow[b]{2}{*}{ 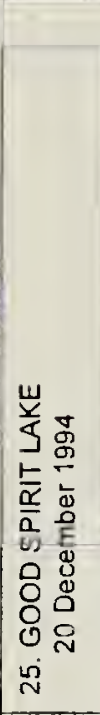 } & \multicolumn{12}{|c|}{ LOCATION AND DATE } \\
\hline & 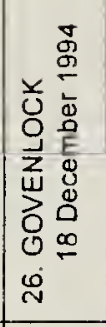 & 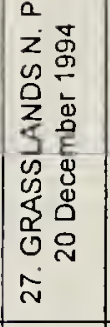 & 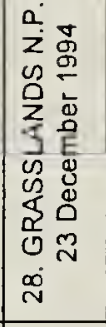 & 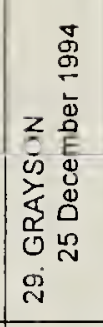 & 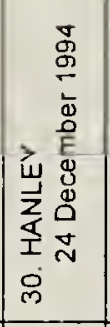 & 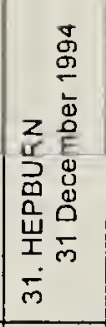 & 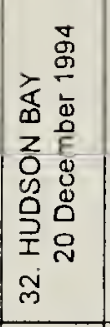 & 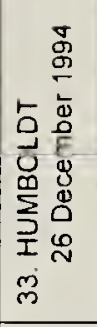 & 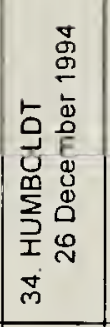 & 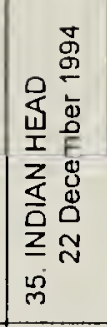 & 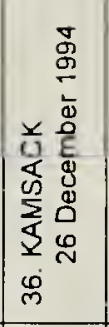 & SPECIES \\
\hline+ & 3 & & & 2 & & & & + & & $\begin{array}{r}56 \\
1 \\
2\end{array}$ & 1 & $\begin{array}{l}\text { CANADA GOOSE } \\
\text { MALLARD } \\
\text { COMMON GOLDENEYE } \\
\text { BALD EAGLE } \\
\text { NORTHERN GOSHAWK } \\
\end{array}$ \\
\hline & $\begin{array}{l}1 \\
5\end{array}$ & $\begin{array}{r}17 \\
1 \\
\\
1 \\
\end{array}$ & 1 & & & & & & & & 1 & $\begin{array}{l}\text { ROUGH-LEGGED HAWK } \\
\text { GOLDEN EAGLE } \\
\text { MERLIN } \\
\text { GYRFALCON } \\
\text { PRAIRIE FALCON } \\
\end{array}$ \\
\hline $\begin{array}{r}1 \\
+ \\
18 \\
\end{array}$ & $\begin{array}{l}13 \\
24 \\
65\end{array}$ & $\begin{array}{r}13 \\
3 \\
3\end{array}$ & 22 & 28 & 2 & & 1 & $\begin{array}{r}6 \\
500 \\
\end{array}$ & $\begin{array}{c}12 \\
2 \\
1\end{array}$ & $\begin{array}{l}11 \\
29 \\
55 \\
\end{array}$ & $\begin{array}{l}20 \\
20\end{array}$ & $\begin{array}{l}\text { GRAY PARTRIDGE } \\
\text { RING-NECKED PHEASANT } \\
\text { RUFFED GROUSE } \\
\text { SHARP-TAILED GROUSE } \\
\text { ROCK DOVE } \\
\end{array}$ \\
\hline $\begin{array}{r}4 \\
9 \\
10\end{array}$ & $\begin{array}{r}16 \\
4\end{array}$ & $\begin{array}{l}1 \\
1\end{array}$ & 1 & $\begin{array}{l}1 \\
1 \\
3 \\
3\end{array}$ & & 2 & & $\begin{array}{l}+ \\
+ \\
2 \\
1\end{array}$ & 1 & $\begin{array}{l}4 \\
3 \\
8 \\
7\end{array}$ & $\begin{array}{r}1 \\
1 \\
11 \\
9\end{array}$ & $\begin{array}{l}\text { GREAT HORNED OWL } \\
\text { SNOWY OWL } \\
\text { DOWNY WOODDPECKER } \\
\text { HAIRY WOODPECKER } \\
\text { THREE-TOED WOODPECKER }\end{array}$ \\
\hline $\begin{array}{l}+ \\
6 \\
\end{array}$ & 248 & 31 & 5 & 1 & & + & 7 & + & & 33 & $\begin{array}{r}11 \\
1 \\
49 \\
\end{array}$ & $\begin{array}{l}\text { NORTHERN FLICKER } \\
\text { PILEATED WOODPECKER } \\
\text { HORNED LARK } \\
\text { GRAY JAY } \\
\text { BLUE JAY } \\
\end{array}$ \\
\hline $\begin{array}{l}24 \\
27 \\
45\end{array}$ & 23 & 34 & 30 & 35 & $\begin{array}{l}1 \\
4 \\
2\end{array}$ & 3 & $\begin{array}{r}13 \\
393 \\
36 \\
11 \\
\end{array}$ & $\begin{array}{r}3 \\
+ \\
19\end{array}$ & $\begin{array}{r}10 \\
4\end{array}$ & $\begin{array}{l}55 \\
10 \\
69 \\
2 \\
\end{array}$ & $\begin{array}{r}23 \\
61 \\
130 \\
1 \\
\end{array}$ & $\begin{array}{l}\text { BLACK-BILLED MAGPIE } \\
\text { COMMON RAVEN } \\
\text { BLACK-CAPPED CHICKADEE } \\
\text { BOREAL CHICKADEE } \\
\text { RED-BREASTED NUTHATCH }\end{array}$ \\
\hline 22 & & & & 4 & 12 & 110 & & 450 & 30 & \begin{tabular}{r|}
4 \\
6 \\
22 \\
121 \\
\end{tabular} & $\begin{array}{r}17 \\
100 \\
40 \\
\end{array}$ & $\begin{array}{l}\text { WHITE-BREASTED NUTHATCH } \\
\text { GOLDEN-CROWNED KINGLET } \\
\text { AMERICAN ROBIN } \\
\text { BOHEMIAN WAXWING } \\
\text { CEDAR WAXWING } \\
\end{array}$ \\
\hline $\begin{array}{l}+ \\
8\end{array}$ & $\begin{array}{r}1 \\
6 \\
1 \\
33 \\
\end{array}$ & $\begin{array}{r}1 \\
30 \\
130 \\
\end{array}$ & & & & & & 6 & 1 & $\begin{array}{r}1 \\
340 \\
\end{array}$ & $\begin{array}{r}33 \\
100 \\
\end{array}$ & $\begin{array}{l}\text { NORTHERN SHRIKE } \\
\text { EUROPEAN STARLING } \\
\text { HARRIS' SPARROW } \\
\text { DARK-EYED JUNCO } \\
\text { SNOWBUNTING } \\
\end{array}$ \\
\hline $\begin{array}{r}4 \\
52 \\
\end{array}$ & 926 & 110 & & 21 & 257 & 6 & $\begin{array}{r}51 \\
1 \\
\end{array}$ & 14 & 6 & 108 & $\begin{array}{r}141 \\
21 \\
40 \\
324 \\
119 \\
\end{array}$ & $\begin{array}{l}\text { RUSTY BLACKBIRD } \\
\text { PINE GROSBEAK } \\
\text { COMMON REDPOLL } \\
\text { PINE SISKIN } \\
\text { EVENING GROSBEAK } \\
\text { HOUSE SPARROW }\end{array}$ \\
\hline 17 & 19 & 17 & 7 & 12 & 6 & 3 & 9 & 13 & 9 & 30 & 25 & NO. SPECIES COUNT DAY \\
\hline 22 & 19 & 17 & 7 & 12 & 6 & 4 & 9 & 18 & 9 & 30 & 25 & NO. SPECIES COUNT PERIOD \\
\hline 285 & 1425 & 391 & 94 & 106 & 278 & 121 & 519 & 1049 & 67 & 958 & 1275 & NO. INDIV. COUNT DAY \\
\hline 0 & 52 & 12 & 3 & 0 & 0 & 0 & 0 & 0 & 0 & 7 & 0 & NO. INDIV. IN TABLE $4 \& 5$ \\
\hline 1 & 2 & 2 & 1 & 0 & 0 & 0 & 0 & 0 & 0 & 5 & 0 & NO. SPECIES IN TABLE 485 \\
\hline
\end{tabular}


Table 3-4. SPECIES RECORDED FROM MORE THAN FIVE LOCALITIES $(+=$ species seen during the count period but not on count day)

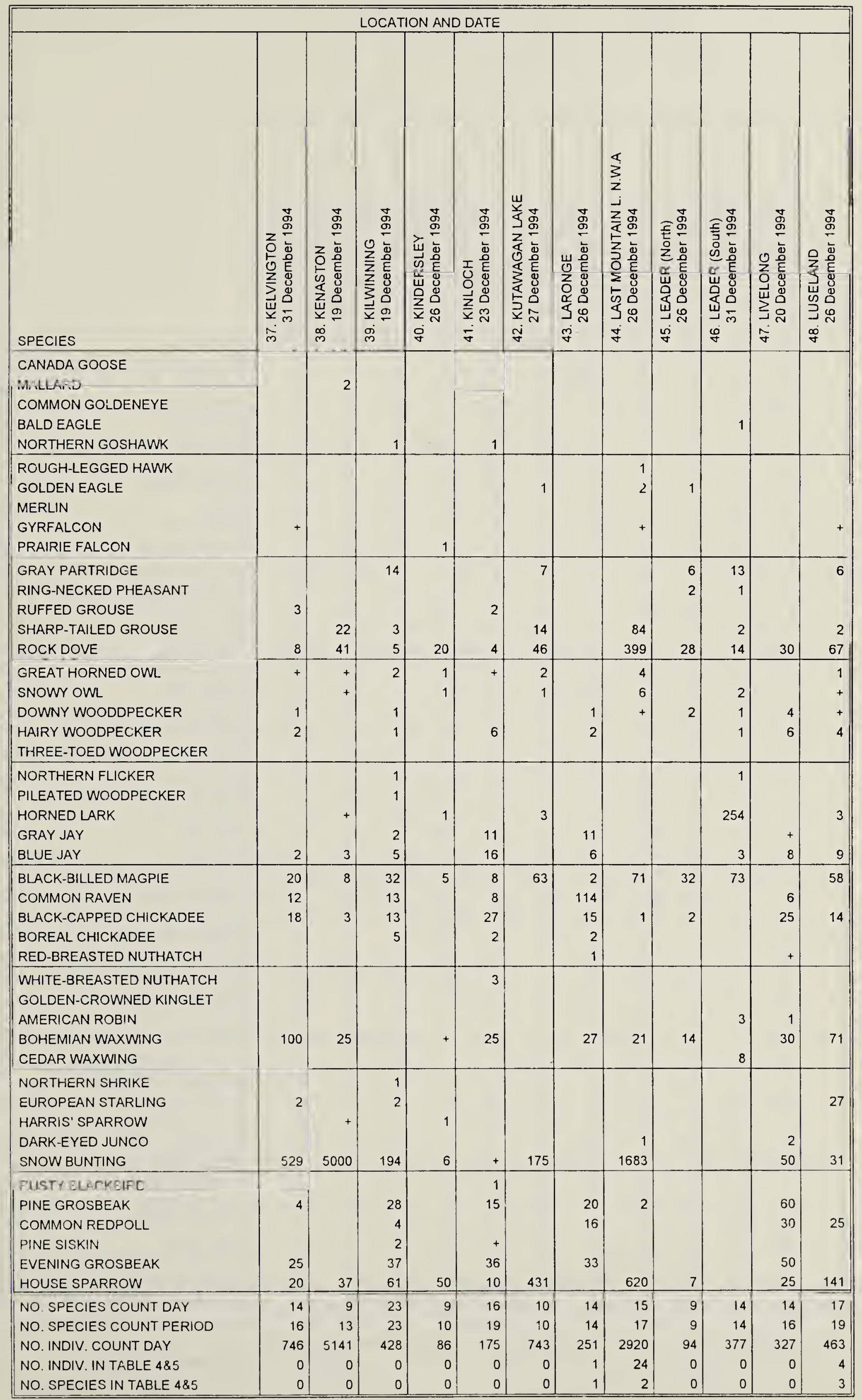


Table 3-5. SPECIES RECORDED FROM MORE THAN FIVE LOCALITIES $(+=$ species seen during the count period but not on count day)

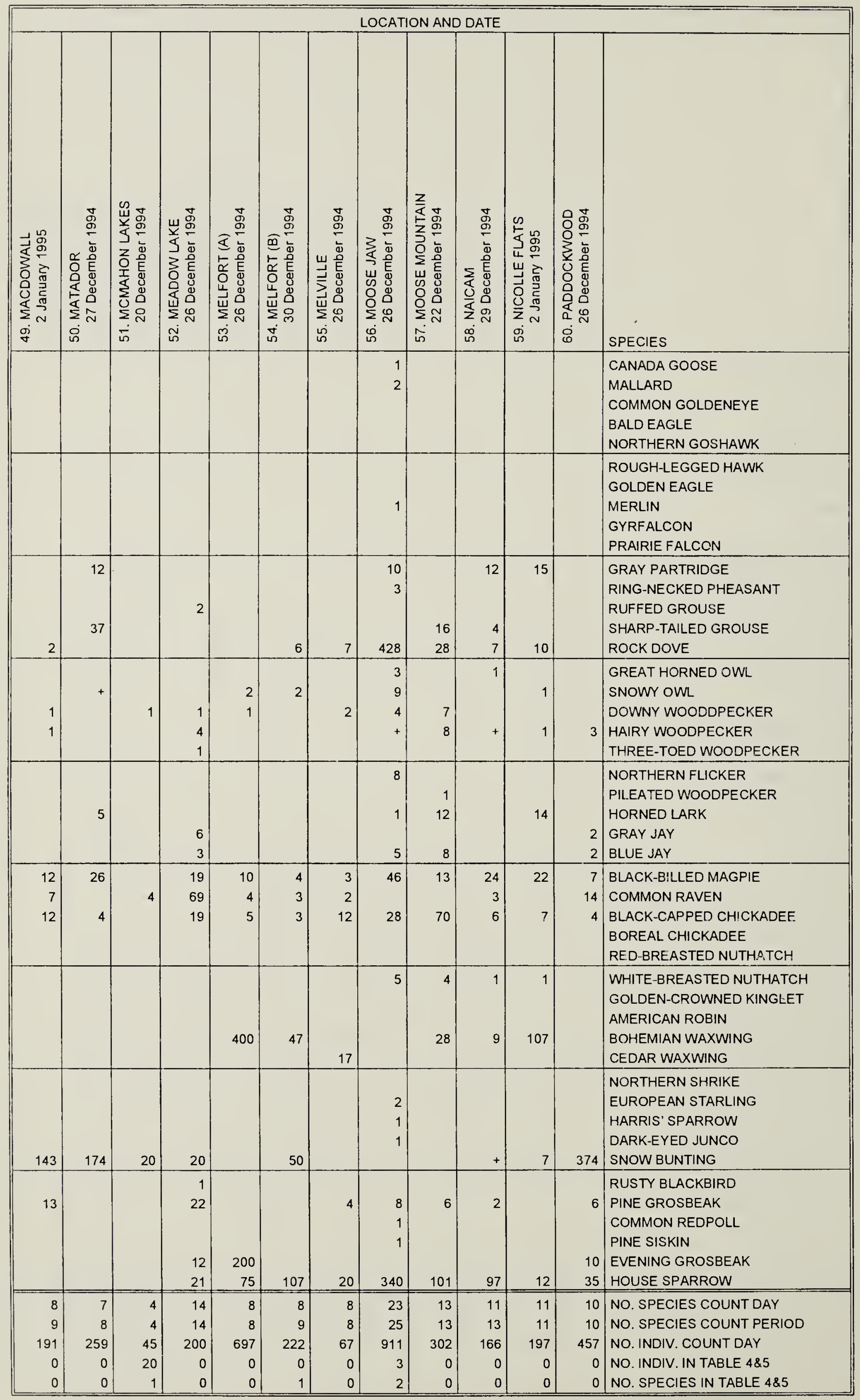


Table 3-6. SPECIES RECORDED FROM MORE THAN FIVE LOCALITIES $(+=$ species seen during the count period but not on count day)

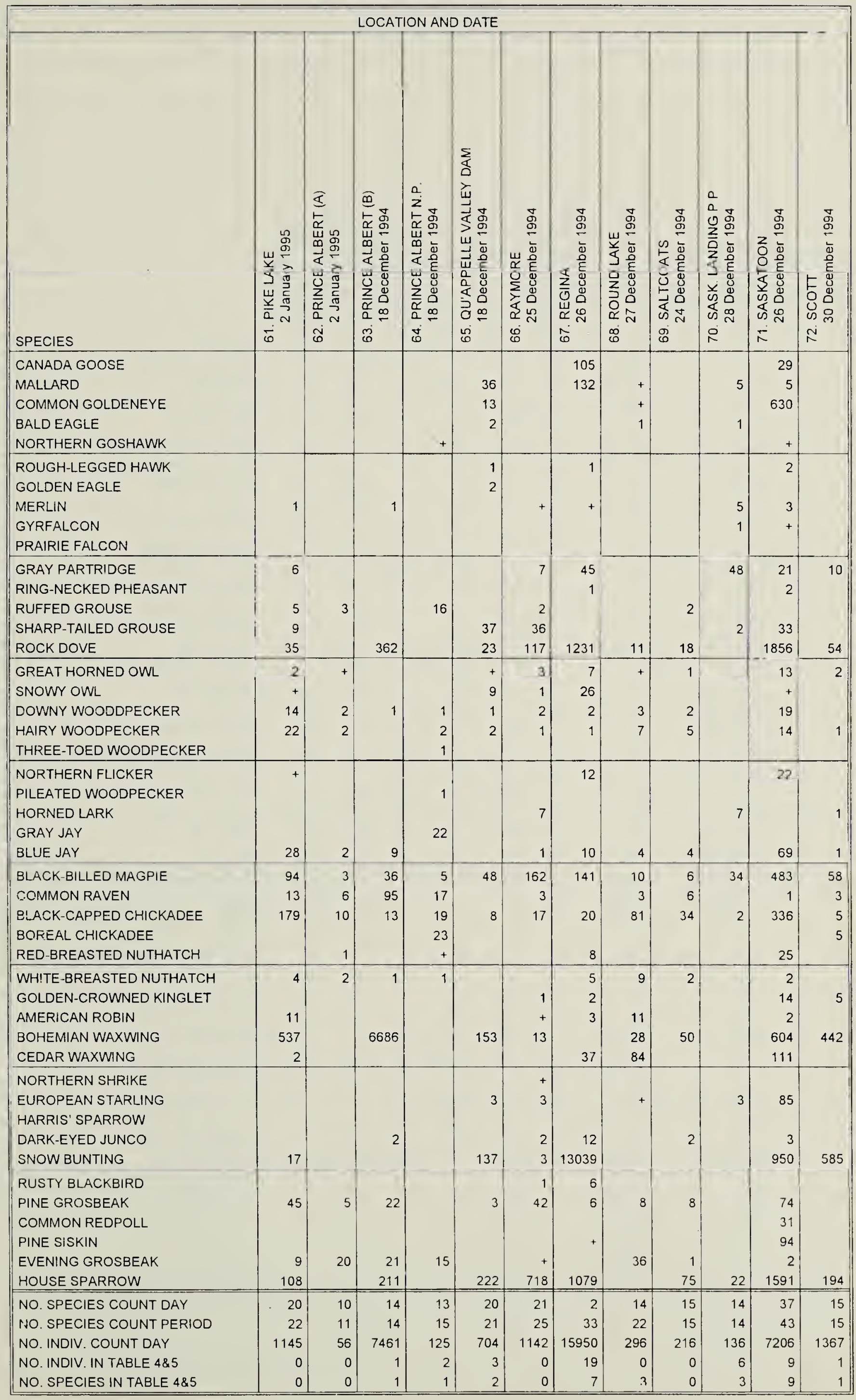


Table 3-7. SPECIES RECORDED FROM MORE THAN FIVE LOCALITIES $(+=$ species seen during the count period but not on count day)

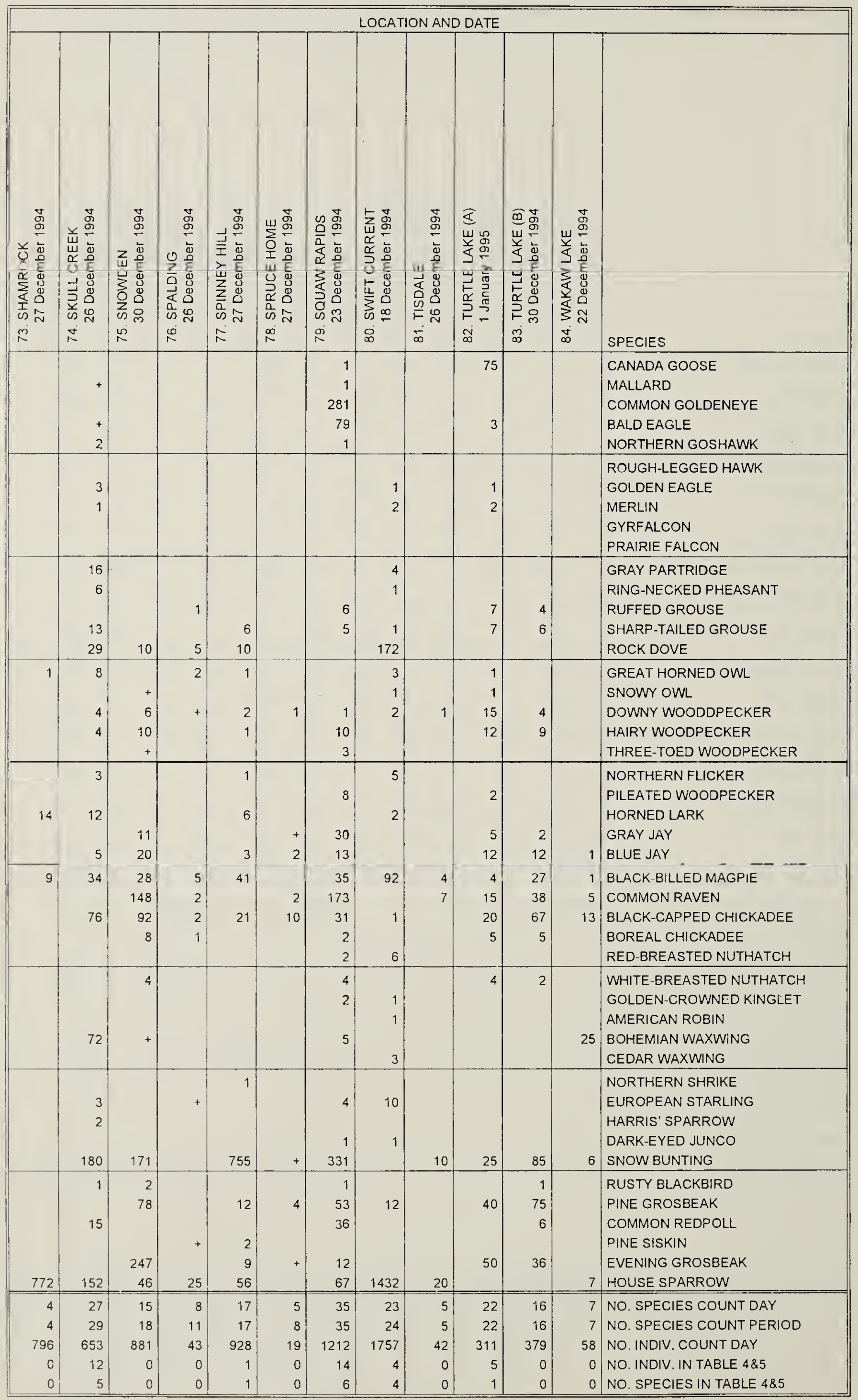


Table 3-8. SPECIES RECORDED FROM MORE THAN FIVE LOCALITIES $(+=$ species seen during the count period but not on count day)

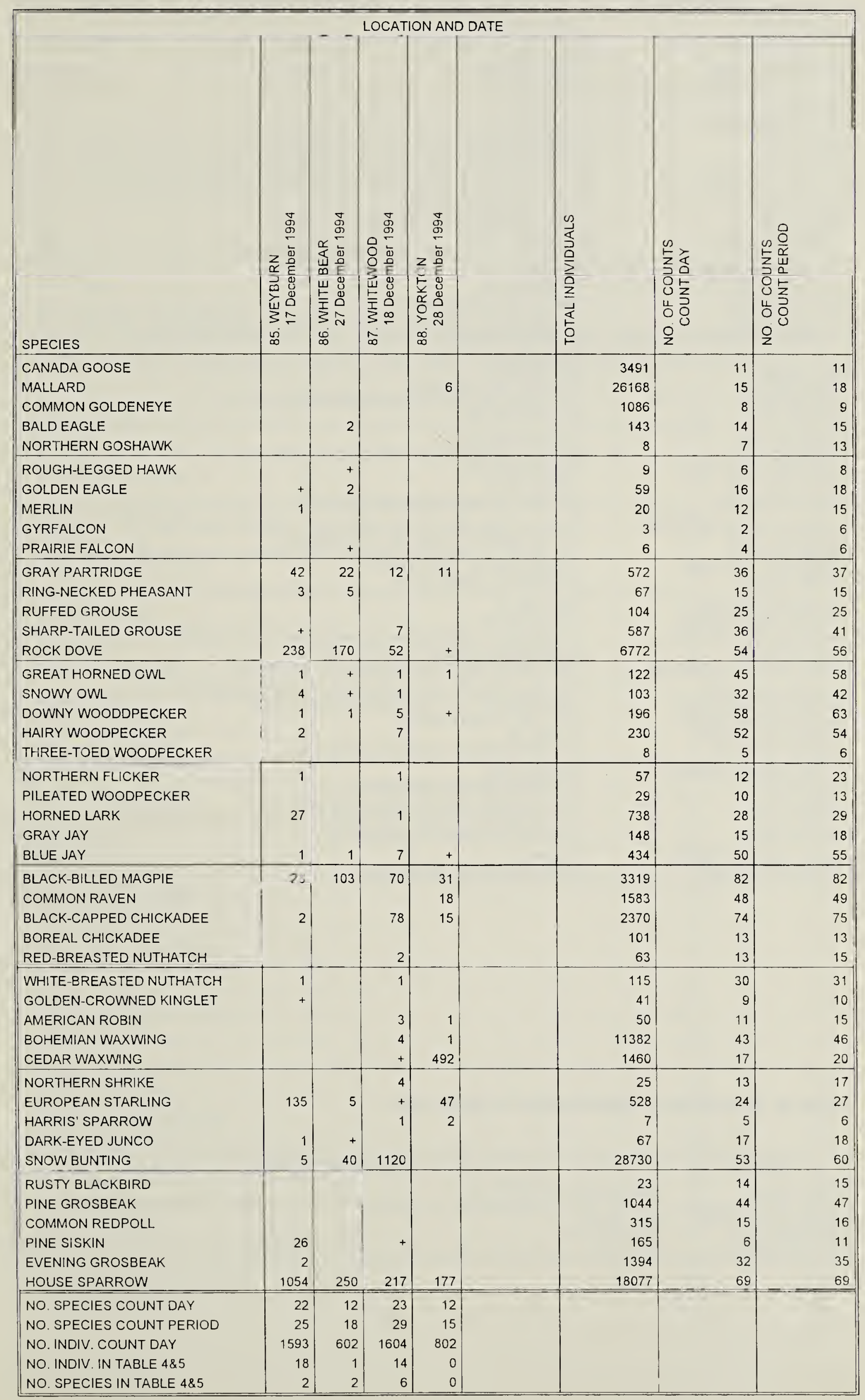


Table 4. SPECIES SEEN ON FIVE OR FEWER COUNTS

\begin{tabular}{|c|c|}
\hline SPECIES & LOCATION AND NUMBER \\
\hline $\begin{array}{l}\text { TUNDRA SWAN } \\
\text { GREEN-WINGED TEAL } \\
\text { AMERICAN BLACK DUCK } \\
\text { NORTHERN PINTAIL } \\
\text { NORTHERN SHOVELER }\end{array}$ & $\begin{array}{l}\text { Indian Head (2) } \\
\text { Fife Lake (1), Qu'Appelle Valley Dam (2), Saskatchewan Landing P.P. (4), Saskatoon (1) } \\
\text { Regina (2) } \\
\text { Fort Qu'Appelle (1) } \\
\text { Crooked Lake (5) }\end{array}$ \\
\hline $\begin{array}{l}\text { GADWALL } \\
\text { AMERICAN WIDGEON } \\
\text { REDHEAD } \\
\text { LESSER SCAUP } \\
\text { BUFFLEHEAD } \\
\end{array}$ & $\begin{array}{l}\text { Coronach (3) } \\
\text { Coronach (2) } \\
\text { Fort Qu'Appelle (1) } \\
\text { Crooked Lake (1), Fort Qu'Appelle (11), Round Lake (+), Squaw Rapids (1) } \\
\text { Coronach (2), Fort Qu'Appelle (1), Indian Head (1), Round Lake (+), Saskatoon (1) }\end{array}$ \\
\hline $\begin{array}{l}\text { COMMON MERGANSER } \\
\text { RED-BREASTED MERGANSER } \\
\text { RUDDY DUCK } \\
\text { TURKEY VULTURE } \\
\text { NORTHERN HARRIER } \\
\end{array}$ & $\begin{array}{l}\text { Fort Qu'Appelle (1), Gardiner Dam (209), Saskatoon (1), Squaw Rapids (5) } \\
\text { Gardiner Dam (1) } \\
\text { Gardiner Dam (1), Squaw Rapids (2) } \\
\text { Crooked Lake (1) } \\
\text { Fife Lake (+), Govenlock (1), Last Mountain Lake W.M.A. (1), Luseland (2) }\end{array}$ \\
\hline $\begin{array}{l}\text { SHARP-SHINNED HAWK } \\
\text { COOPER'S HAWK } \\
\text { RED-TAILED HAWK } \\
\text { SPRUCE GROUSE } \\
\text { WILLOW PTARMIGAN } \\
\end{array}$ & $\begin{array}{l}\text { White Bear }(+) \\
\text { Indian Head (1) } \\
\text { Melfort }(B)(+) \text {, Moose Jaw }(+) \\
\text { Candle Lake (4), Christopher Lake }(+) \text {, La Ronge (1), Squaw Rapids (4), Turtle Lake (A) (5) } \\
\text { McMahon Lakes (20) }\end{array}$ \\
\hline $\begin{array}{l}\text { SAGE GROUSE } \\
\text { WILD TURKEY } \\
\text { AMERICAN COOT } \\
\text { COMMON SNIPE } \\
\text { HERRING GULL } \\
\end{array}$ & $\begin{array}{l}\text { Govenlock (51) } \\
\text { Fort Walsh (1) } \\
\text { Coronach (23), Fife Lake (3) } \\
\text { Qu'Appelle Valley Dam (1), Saskatchewan Landing P.P. (1) } \\
\text { Gardiner Dam (3) }\end{array}$ \\
\hline $\begin{array}{l}\text { GLAUCOUS GULL } \\
\text { MOURNING DOVE } \\
\text { NORTHERN HAWK-OWL } \\
\text { GREAT GRAY OWL } \\
\text { SHORT-EARED OWL } \\
\end{array}$ & $\begin{array}{l}\text { Gardiner Dam (1) } \\
\text { Regina (+), Skull Creek (1), Swift Current (2), White Bear (1) } \\
\text { Squaw Rapids (1) } \\
\text { Whitewood (+) } \\
\text { Fife Lake }(+) \text {, Last Mountain Lake W.M.A. (23) }\end{array}$ \\
\hline $\begin{array}{l}\text { BLACK-BACKED WOODPECKER } \\
\text { AMERICAN CROW } \\
\text { BROWN CREEPER } \\
\text { TOWNSEND'S SOLITAIRE } \\
\text { VARIED THRUSH }\end{array}$ & $\begin{array}{l}\text { Armit (1), Prince Albert N.P. (2), Saskatoon (+), Scott (1), Squaw Rapids (1) } \\
\text { Fort Qu'Appelle (+), Moose Jaw (3), Saskatoon (1) } \\
\text { Fort Walsh (1), Luseland (1), Regina (2), Swift Current (1), Whitewood (1) } \\
\text { Gardiner Dam (1), Swift Current (1) } \\
\text { Fort Walsh (1), Whitewood (+) }\end{array}$ \\
\hline $\begin{array}{l}\text { LOGGERHEAD SHRIKE } \\
\text { RUFOUS-SIDED TOWHEE } \\
\text { AMERICAN TREE SPARROW } \\
\text { SONG SPARROW } \\
\text { WHITE-THROATED SPARROW }\end{array}$ & $\begin{array}{l}\text { Fife Lake (+) } \\
\text { Skull Creek (1) } \\
\text { Fort Walsh (11), Gardiner Dam (2), Indian Head (2), Skull Creek (8) } \\
\text { Saskatchewan Landing P.P. (1), Skull Creek (1) } \\
\text { Birch Hills (1), Indian Head (1), Regina (1), Saskatoon (1) }\end{array}$ \\
\hline $\begin{array}{l}\text { LAPLAND LONGSPUR } \\
\text { RED-WINGED BLACKBIRD } \\
\text { WESTERN MEADOWLARK } \\
\text { BREWER'S BLACKBIRD } \\
\text { COMMON GRACKLE } \\
\end{array}$ & $\begin{array}{l}\text { Bromhead (35), Fife Lake (+), Grasslands N.P. (1), Grasslands N.P. (NW) (3) } \\
\text { Grasslands N.P. (11), Swift Current (+), Whitewood (3) } \\
\text { Fife Lake (+), Skull Creek (1) } \\
\text { Craven (2) } \\
\text { Fort Qu'Appelle (1), Luseland (1), Weyburn (2) }\end{array}$ \\
\hline $\begin{array}{l}\text { BROWN-HEADED COWBIRD } \\
\text { ROSY FINCH } \\
\text { PURPLE FINCH } \\
\text { HOUSE FINCH } \\
\text { RED CROSSBILL } \\
\end{array}$ & $\begin{array}{l}\text { Whitewood (6) } \\
\text { Fort Walsh (16), Good Spirit Lake (+) } \\
\text { Fort Qu'Appelle (1), Round Lake (+), Spinney Hill (1) } \\
\text { Fort Qu'Appelle (+), Regina (14), Saskatoon (+), Weybum (16), Whitewood (4) } \\
\text { Fort Walsh (2), Regina (+), Saskatoon (4) }\end{array}$ \\
\hline $\begin{array}{l}\text { WHITE-WINGED CROSSBILL } \\
\text { AMERICAN GOLDFINCH }\end{array}$ & $\begin{array}{l}\text { Regina (+) } \\
\text { Prince Albert (B) (1), Saskatoon (+) }\end{array}$ \\
\hline
\end{tabular}

Table 5. BIRDS NOT IDENTIFIED TO SPECIES

\begin{tabular}{|l|l|}
\hline \multicolumn{1}{|c|}{ SPECIES } & \multicolumn{1}{|c|}{ LOCATION AND NUMBER } \\
\hline EAGLE species & Last Mountain Lake W.M.A. (1) \\
LARGE FALCON species & Gardiner Dam (1), Grasslands N.P. (1), Grasslands N.P. (NW) (1), Matador (1), Qu'Appelle Valley Dam (1) \\
WOODPECKER species & Pike Lake (4) \\
WAXWING species & Saskatoon (71) \\
REDPOLL species & Craven (2) \\
\hline
\end{tabular}


Table 6. SUMMARY OF NEW OR TYING HIGH COUNTS ESTABLISHED DURING 1994 COUNTS (tying counts regular type; boldface/italic indicates a new record)

\begin{tabular}{|c|c|c|c|c|}
\hline LOCATION & $\begin{array}{c}1993 \\
\text { COUNT }\end{array}$ & $\begin{array}{c}\text { SPECIES } \\
\text { COUNT }\end{array}$ & $\begin{array}{c}\text { PREVIOUS } \\
\text { HIGH }\end{array}$ & LOCATION AND YEAR \\
\hline $\begin{array}{l}\text { Regina } \\
\text { Coronach } \\
\text { Coronach } \\
\text { Squaw Rapids } \\
\text { Luseland }\end{array}$ & $\begin{array}{r}2 \\
15150 \\
3 \\
79 \\
2\end{array}$ & $\begin{array}{l}\text { Am. Black Duck } \\
\text { Mallard } \\
\text { Gadwall } \\
\text { Bald Eagle } \\
\text { Northern Harrier }\end{array}$ & $\begin{array}{r}2 \\
10000 \\
2 \\
52 \\
1\end{array}$ & $\begin{array}{l}\text { Regina '92 } \\
\text { Fife Lake '90 } \\
\text { Regina '61 } \\
\text { Squaw Rapids '81 } \\
\text { several counts } n \text {. to Harris, Hawarden }\end{array}$ \\
\hline $\begin{array}{l}\text { Kamsack } \\
\text { Hudson Bay } \\
\text { Fort Walsh } \\
\text { Skull Creek } \\
\text { Prince Albert }\end{array}$ & $\begin{array}{r}11 \\
393 \\
1 \\
1 \\
1\end{array}$ & $\begin{array}{l}\text { Pileated Woodpecker } \\
\text { Common Raven } \\
\text { Varied Thrush } \\
\text { Rufous-sided Towhee } \\
\text { American Goldfinch }\end{array}$ & $\begin{array}{r}4 \\
279 \\
1 \\
1 \\
1\end{array}$ & $\begin{array}{l}\text { Kamsack '93 } \\
\text { Nipawin' } 93 \\
\text { n. to Saskatoon } \\
\text { Moose Jaw '63, Fort Qu'Appelle '93, Leader '93 } \\
\text { Saskatoon '79, Regina '81, Craven '93 }\end{array}$ \\
\hline
\end{tabular}

Table 7. COMPARISON OF THE 1994 POPULATIONS TO 1993 AND AVERAGE PAST YEARS (1989-1993) BASED ON PARTY HOURS PER INDIVIDUAL

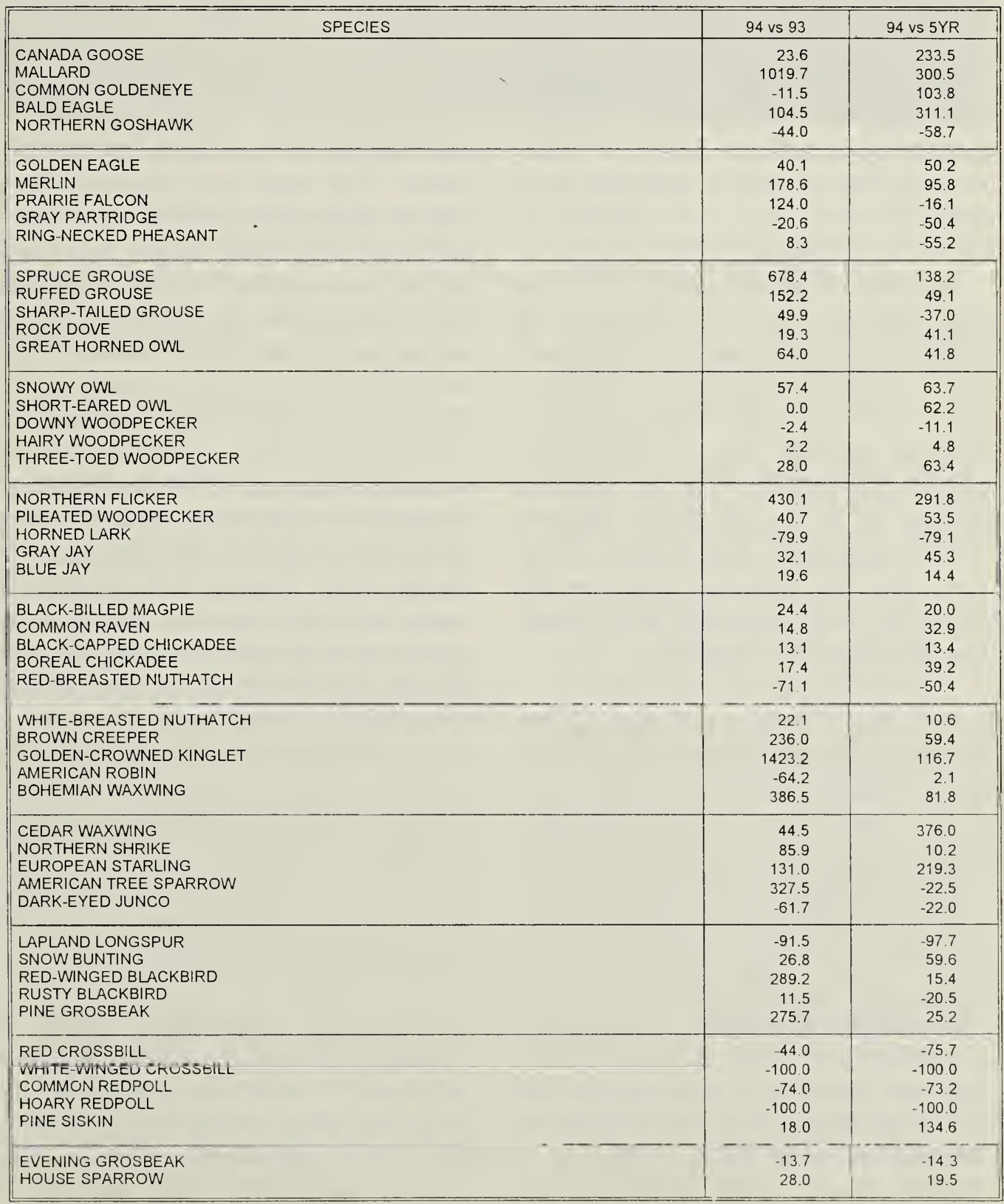

